\title{
Neuropsychological Measures that Predict Progression from Mild Cognitive Impairment to Alzheimer's type dementia in Older Adults: a Systematic Review and Meta-Analysis
}

\author{
Sylvie Belleville ${ }^{1,2} \cdot$ Céline Fouquet $^{1} \cdot$ Carol Hudon ${ }^{3,4}$. \\ Hervé Tchala Vignon Zomahoun ${ }^{5,6}$. Jordie Croteau ${ }^{5,6}$ • Consortium for the Early \\ Identification of Alzheimer's disease-Quebec
}

Received: 21 November 2016 / Accepted: 4 September 2017 / Published online: 10 October 2017

(C) The Author(s) 2017. This article is an open access publication

\begin{abstract}
This study aimed to determine the extent to which cognitive measures can predict progression from mild cognitive impairment (MCI) to Alzheimer's type dementia (AD), assess the predictive accuracy of different cognitive domain categories, and determine whether accuracy varies as a function of age and length of follow-up. We systematically reviewed and meta-analyzed data from longitudinal studies reporting sensitivity and specificity values for neuropsychological tests to identify individuals with MCI who will develop AD. We searched articles in Medline, Cochrane, EMBASE, PsycINFO, and the Web of Science. Methodological quality was assessed using the STARDem and QUADAS standards. Twenty-eight studies met the eligibility criteria (2365 participants) and reported predictive values from 61 neuropsychological tests with a 31-month mean follow-up. Values were pooled to provide combined accuracy for 14 cognitive domains. Many domains showed very good predictive accuracy with high sen-
\end{abstract}

Sylvie Belleville, Céline Fouquet and Carol Hudon are part of Consortium for the Early Identification of Alzheimer's disease-Quebec (CIMA-Q).

Electronic supplementary material The online version of this article (https://doi.org/10.1007/s11065-017-9361-5) contains supplementary material, which is available to authorized users.

Sylvie Belleville

sylvie.belleville@umontreal.ca

1 Research Center of the Institut Universitaire de Gériatrie de Montréal, 4565 Chemin Queen Mary, Montréal, Québec H3W 1W5, Canada

2 Université de Montréal, CP 6128 Succ. Centre Ville, Montréal, Québec H3C-1J7, Canada

3 Université Laval, Pavillon Félix-Antoine-Savard, 2325, rue des Bibliothèques, Local 1546, Québec, Québec G1V 0A6, Canada sitivity and specificity values $(\geq 0.7)$. Verbal memory measures and many language tests yielded very high predictive accuracy. Other domains (e.g., executive functions, visual memory) showed better specificity than sensitivity. Predictive accuracy was highest when combining memory measures with a small set of other domains or when relying on broad cognitive batteries. Cognitive tests are excellent at predicting MCI individuals who will progress to dementia and should be a critical component of any toolkit intended to identify $\mathrm{AD}$ at the predementia stage. Some tasks are remarkable as early indicators, whereas others might be used to suggest imminent progression.

Keywords Alzheimer's disease · Mild cognitive impairment . Neuropsychology $\cdot$ Diagnosis $\cdot$ Cognitive tests $\cdot$ Predictive accuracy
4 CERVO Brain Research Center, 2601, de la Canardiere, Québec, Québec G1J 2G3, Canada

$5 \quad$ Health and Social Services Systems, Knowledge Translation and Implementation component of the Quebec SPOR-SUPPORT Unit, Université Laval, Québec, Québec G1L 2E8, Canada

6 Population Health and Practice-Changing Research Group, Research Centre of CHU de Québec- Université Laval, Québec, Québec G1L 2E8, Canada 


\section{Introduction}

Alzheimer's disease (AD) is progressive. It is only diagnosed with certainty when the post-mortem neuropathological examination reveals the presence of amyloid plaques and neurofibrillary tangles in the brains of patients who have suffered from the clinical symptoms prior to their death. Anteromortem diagnosis thus relies on a set of clinical inclusion and exclusion criteria (e.g., APA 2013; McKhann et al. 2011). Based on current criteria, the diagnosis of probable $\mathrm{AD}$ is given when patients have dementia which is defined as cognitive impairment that involves at least two cognitive domains and is sufficiently severe that it interferes with activities of daily living (APA 2013; McKhann et al. 2011). However, dementia corresponds to the end phase of AD. Studies on patients suffering from the autosomal dominant version of the disease and population studies that have followed patients or analyzed their performance retrospectively, suggest that the disease probably starts $10-15$ years earlier than when patients typically receive their diagnosis (Amieva et al. 2008; Bateman et al. 2012). This late diagnosis considerably impedes research as it limits the ability to identify the early mechanisms that trigger the disease and contribute to its progression. Furthermore, a considerable effort has been devoted to finding a curative treatment and identifying effective lifestyle prevention strategies for AD. When discovered, such treatment or preventative approaches will have to be provided before the disease has produced major damage to the brain to have the most benefit. These issues have motivated a substantial research effort towards detection at an earlier stage of the disease, prior to dementia.

Mild cognitive impairment (MCI) has been of particular interest in this regard. Individuals with MCI complain about their memory and show mild cognitive deficits when tested with objective cognitive measures (Petersen 2000; Gauthier et al. 2011; Albert et al. 2011), but their cognitive impairment is not sufficiently severe to meet the criteria for dementia. Yet, these individuals have a ten-fold greater risk of progression to dementia than the general population, suggesting that many are actually in a pre-dementia phase of the disease (Gauthier et al. 2011; Petersen 2000). The cognitive assessment performed by neuropsychologists is a central component for the diagnosis of $\mathrm{AD}$ at the dementia phase, and it is thus critical to have access to sensitive and specific cognitive measures. Relying on appropriate measures is even more critical during the MCI phase, in which cognitive impairment is mild and functional impact minimal. Differentiating the cognitive deficits related to incipient $\mathrm{AD}$ from those related to cognitive fluctuations due to normal aging or other non-morbid conditions is particularly challenging, because impairment is mild at this phase.

Many studies have characterized the cognition of people with MCI relative to that of healthy older adults using cross- sectional designs (for reviews, see Belleville et al. 2008; Belleville et al. 2014a). These studies have provided clinicians with cognitive characterization of this early phase and clinical tools that can be used to determine whether older adults meet the criteria for MCI. However, not all individuals who meet the criteria for MCI will progress to dementia and one major challenge is to identify tests that differentiate between individuals with MCI who will remain stable and those who will progress toward dementia. Many studies have attempted to identify early markers of future progression. These studies rely on a longitudinal design in which MCI participants are tested at entry on a set of cognitive measures and followed over time to identify progressors versus non-progressors. Regression analyses can then be used to assess whether performance on cognitive tests completed at entry indeed predict clinical progression.

Many of these prognostic studies relied on comparable tests and it is therefore possible to pool the data to perform quantitative meta-analyses. This allows us to address many important questions related to early cognitive diagnosis. One major question is whether cognitive tests fare well in predicting progression from $\mathrm{MCI}$ to dementia, which is crucial in a context where much effort is devoted to developing biomarkers of progression. Cognitive assessments are widely available and relatively low-cost. Thus, it is important to determine their predictive accuracy and provide quantitative values so that they can be compared to more costly biomarkers. Previous reviews have indicated that the predictive accuracy reported in the literature is extremely variable (Belleville et al. 2014a). This could be due to a wide range of factors, including the cognitive domain measured by the task, length of follow-up, or the characteristics of the patient. Tests might also differ in terms of their specificity and sensitivity. In prognostic studies, sensitivity refers to the correct identification of progressors, whereas specificity refers to the correct rejection of non-progressors.

One important question is to what extent different domains determine overall predictive accuracy, sensitivity, and specificity. For example, some of the most well-accepted clinical criteria have proposed that cued recall (Dubois et al. 2007) or delayed memory (Albert et al. 2011) may be the most highly affected in MCI. It is thus important to assess whether this is the case by conducting an appropriate meta-analysis of the data. Another issue is the role of non-memory domains in early diagnosis. Some studies have found that working memory, executive functions, and language can be impaired in MCI (Belleville et al. 2007; Joubert et al. 2008; Saunders and Summers 2010). However, these studies were based on cross-sectional group comparisons and it is unclear whether these tests are sufficiently sensitive to predict dementia at the individual level. In contrast, non-memory-based tests may have value for specificity, because impairment of nonmemory aspects may be more problematic when present. A 
few studies have examined multiple tests or those that cover a wide range of cognitive functions as predictors of dementia. This strategy may optimize accuracy by pooling tests with a different balance between their sensitivity and specificity (Summers and Saunders 2012; Belleville et al. 2014b).

A related question is the impact that age might have on predictive values of different tests. Age is a potentially important factor because aging itself is accompanied by a specific set of cognitive deficits and because mixed dementia is more frequent in older patients. Thus, older age might be associated with a larger contribution from non-memory domains, particularly executive functions. The length of follow-up is a major issue as well. Studies with shorter follow-ups run the risk of identifying an individual as stable even though they would have progressed to dementia had there been a longer followup. Thus, shorter follow-ups increase the likelihood of false negatives and might reduce test sensitivity for the task being evaluated, whereas a longer follow-up should increase sensitivity. A sufficiently long follow-up can be used as a proxy for how far the patient was from the diagnosis when the test was given. Thus, assessing the impact of follow-up length is also informative for identifying whether a task is more useful as a very early marker or as a reflection of imminent progression. A task found to be sensitive at a longer follow-up might be particularly well suited as an early indicator of future dementia. Thus, studies with longer average follow-ups might identify earlier predictors. In turn, a task with a relatively low sensitivity at longer follow-ups but with an excellent value at shorter ones would reflect imminent progression.

Our goal was to conduct a systematic review and metaanalysis to determine the extent to which cognitive measures predict progression from MCI to dementia of the AD type. We used data from longitudinal studies reporting sensitivity and specificity values for cognitive tests to identify individuals with MCI who will progress to a diagnosis of AD in the future. The present study does not replicate or update existing reviews. Other authors have systematically reviewed the diagnostic performance of cognitive tests to cross-sectionally identify people with MCI (Ozer et al. 2016) or dementia (Tsoi et al. 2015), and one study has examined which screening tools best predict progression to dementia in primary care patients (Lischka et al. 2012). However, no review has yet investigated the sensitivity and specificity of a variety of cognitive tests to predict progression to AD among individuals with MCI.

The specific questions addressed in this meta-analysis and systematic review are 1) the predictive value of cognitive measures to predict future progression from MCI to dementia and more specifically, their global (or average) sensitivity (correct identification of progressors) and specificity (correct rejection of non-progressors), 2) the impact of age on predictive accuracy, 3 ) the average length of follow-up from reported studies and its impact on predictive accuracy, and 4) the benefits of combining different cognitive domains.

\section{Materials and Methods}

This systematic review was reported in accordance with the PRISMA statement (Liberati et al. 2009). The protocol was not registered, but it was predetermined, and PICOS statements were used to identify the studies to be included in the review and meta-analyses.

\section{Eligibility Criteria}

Criteria for including or excluding articles were determined a priori. The PICOS approach was adapted to formulate the research question. Studies were included if they were longitudinal and designed to assess the sensitivity and specificity of neuropsychological tests to determine future progression from mild cognitive deficit to dementia of the Alzheimer type. Operational inclusion criteria were the following i) articles written in English or French, ii) studies using a prospective or retrospective longitudinal design, iii) at baseline, participants were identified as older adults with MCI and no dementia or a related terminology, iv) neuropsychological or cognitive tests were used as predictors and were sufficiently well described to allow replication, v) participants had a follow-up of at least 1 year, vi) the outcome was a diagnosis of dementia of the Alzheimer disease's type based on the DSM-III-R criteria (APA 1987), DSM-IV criteria (APA 1994) or National Institute of Neurological and Communication Disorders and Stroke/Alzheimer's Disease and Related Disorders Association (NINCDS-ADRDA) criteria (McKhann et al. 1984), and supported by a medical examination or a medical consensus panel, and vii) absolute values for false positives, false negatives, true positives, and true negatives were reported or $2 \times 2$ tables could be constructed based on reported data. No publication date restriction was imposed.

There were also several exclusion criteria. Studies were excluded i) if the diagnosis of AD was based on a single neuropsychological test or if there was no definition of the criteria used for the diagnosis of $\mathrm{AD}$, ii) if the study targeted populations with other neurological diseases or neurodevelopmental disorders (e.g., HIV associated dementia, Parkinson's disease, Down Syndrome), iii) if it used a cross-sectional design and iv) if there were insufficient methodological details to allow replication. Furthermore, only the more recent data were included if the same population sample was used in more than one study.

\section{Information Sources and Search}

A computer-based search was performed from five electronic databases: MEDLINE using PubMed (1946 - Present), Embase using OVID (1947 - Present), Cochrane (1918 Present), PsycINFO (1967 - Present), and Web of Science. The last search was carried out on January 29th, 2014. In 
addition, the reference lists from articles of interest were searched for additional references. The full electronic search strategy for MEDLINE is included in Appendix A (Supplementary material). The search strategy, based on the PICOS approach, was applied through a combination of five concepts that delimited our research question 1) Patient, defined as "elderly population" with "MCI", 2) Intervention, defined as the index tests, that is, the different neuropsychological predictors, 3) Comparison, defined by the type of analysis expected in the selected studies i.e., those including predictive values for the neuropsychological predictors, 4) Outcome, defined as the predicted outcome which was "Alzheimer's Disease" and 5) Type of study, which specified that "longitudinal studies" were the type of studies sought. The search strategy was reviewed and validated by a librarian who is an expert in database searches.

\section{Study Selection}

The articles produced by the search strategy were first screened based on the titles and then selected by one reviewer (CF) based on the abstracts. One additional reviewer ( $\mathrm{CH}$ or $\mathrm{SB}$ ) independently revised the list of potential articles based on the abstracts. The full text of the articles considered to be potentially eligible was then evaluated in detail by the same reviewer for quality assessment and any unresolved issues were discussed with SB. All articles meeting eligibility criteria were included in the qualitative synthesis. Articles reporting data that could be appropriately pooled were included in the meta-analyses: this was done when at least three studies reported data for the same cognitive domain.

\section{Data Collection Process and Data Items}

Two reviewers independently extracted data from all eligible studies using a predetermined form (standardized grid). Disagreements between the two reviewers were resolved by consensus or a third reviewer (CH or SB). The data collected from each manuscript included information on the general description of the study, participants, methodological features, results, analysis, and analytic procedure. Table S1 (Supplementary material) shows the items for which information was collected for all papers.

\section{Risk of Bias in Individual Studies}

The methodological quality and the risk of bias of each study was assessed using the Quality Assessment of Diagnostic Accuracy Studies (QUADAS) tool (Whiting et al. 2003), as suggested by Cochrane guidelines (Reitsma et al. 2009). A tailored quality assessment grid was constructed to apply the quality assessment criteria proposed in QUADAS 2 (Whiting et al. 2011) adapted to prognostic studies (see Table S2 in
Supplementary material). Study quality was assessed by a single reviewer with verification by a second reviewer for i) patient selection and population source (e.g., whether the included sample was representative of the population and whether exclusion criteria were too restrictive), ii) study design (e.g., whether it was a prospective or retrospective study and whether the investigator in charge of diagnosis at followup was blind to the baseline performance), iii) flow and follow-up (e.g., length of follow-up), iv) reference standard and outcome (i.e., adequate procedure for diagnosis at follow-up and absence of partial verification bias), v) index tests and prognosis (i.e., adequate description of the neuropsychological battery), and vi) analysis (e.g., whether the cut-off used for calculation of sensitivity and specificity measures was determined using independent norms). This quality assessment allowed us to classify studies as having a low (L), high $(\mathrm{H})$, or low-high $(\mathrm{LH})$ risk of bias.

\section{Analyses}

Articles reporting appropriate data were included in the metaanalyses. If the prevalence was not provided, it was calculated by dividing the number of participants who had progressed at follow-up by the total number of participants at follow-up. If the number of true positives (TP), false positives (FP), true negatives (TN), and false negatives (FN) were not available, they were calculated based on the values of sensitivity and specificity using the following formula: $\mathrm{TP}=$ sensitivity $\mathrm{x}$ number of progressors, $\mathrm{TN}=$ specificity $\mathrm{x}$ number of nonprogressors, $\mathrm{FN}=$ number of progressors minus $\mathrm{TP}$, $\mathrm{FP}=$ number of non-progressors minus $\mathrm{TN}$.

\section{Variables of Interest}

The first aim of the meta-analyses was to obtain global estimates of predictive accuracy for various categories of neuropsychological tests. The study setting was very similar to that of diagnostic test accuracy studies, except that instead of comparing the diagnostic index test results to a gold standard, the index tests were compared to the diagnostic gold standard performed after a certain follow-up period. We therefore used the same methods as for diagnostic test accuracy metaanalyses by obtaining the global sensitivity and specificity for each test category. Sensitivity refers here to the ability of the test to identify MCI patients who will later progress to dementia or more precisely, whether progressors were impaired on the test. Specificity refers to the ability of the test to identify those who will remain stable, that is, whether stable MCI were unimpaired on the test. An ideal test should have $100 \%$ sensitivity and $100 \%$ specificity, which would result in perfect predictive accuracy. Scores higher than 0.7 are 
typically considered to be very good, whereas scores above 0.5 indicate good classification (Haynes et al. 2006).

Tests were grouped within 22 cognitive domain categories for systematic review (see Table 3), given the large variety of neuropsychological tests used in the various studies. The classification of tests within cognitive domain categories was performed following study selection, but prior to data analysis, by consensus between the authors and validated by the CIMA-Q cognition expert group, which comprises 12 clinicians and researchers with expertise in the neuropsychology of dementia. This led to the identification of five broad domains corresponding to cognitive components that are wellrecognized and believed to depend on distinct neuroanatomical substrates: verbal episodic memory, visual episodic memory, language, executive functions and working memory, and visuo-constructive functions. An additional category was used to include brief and global measures of cognition. We then identified test categories that were judged to reveal different brain or cognitive mechanisms within each domain, or to be differentially affected by dementia. For example, we distinguished immediate from delayed recall, because delayed recall is generally believed to reflect the consolidation processes that occurs within the hippocampus to a larger extent than immediate recall. We distinguished studies using oriented encoding, where the examiner provides cues during the encoding phase, as well as studies using cued recall, where the examiner provides cues during the retrieval phase, because some have argued that providing cues at encoding or retrieval reduces the contribution of executive attention to memory performance.

We separately analyzed studies that identified a combination of tests predicting progression in the systematic review, but they were not included in the meta-analysis. Each cognitive domain category was meta-analyzed separately. The minimum number of studies required to perform a diagnostic accuracy meta-analysis is three (HIQA - IRELAND, IQWiG GERMANY 2015). The 14 domains that met this criterion were the following 1) among verbal memory tests, verbal immediate recall, paragraph delayed recall, word-list free delayed recall with non-oriented encoding, word-list free delayed recall with oriented encoding, word-list cued delayed recall with oriented encoding, and word-list recognition 2) among visual memory tests, delayed recall 3) among language tests, naming tests, tests of semantic knowledge, and semantic fluency 4) among executive tests, switching tests and working memory tests, 5) among visuo-constructive measures, visuospatial tests and visuo-constructive tasks and 6) global measures.

\section{Meta-Analysis}

The Cochrane collaboration and others (Macaskill et al. 2010; HIQA - IRELAND, IQWiG - GERMANY 2015; Dahabreh et al. 2012) strongly recommend the use of the random effects hierarchical bivariate models called bivariate (Reitsma et al. 2005) and hierarchical summary receiver operating characteristic (HSROC: Rutter and Gatsonis 2001) for diagnostic test accuracy meta-analyses. The bivariate and HSROC models are equivalent under most common circumstances, for example when no covariate is included or when the sensitivity (Se) and specificity ( $\mathrm{Sp}$ ) of the bivariate model are adjusted for the same covariates. We used only the bivariate model in our analyses due to software implementation technicalities. There are three main reasons to favor a random effects hierarchical bivariate model (Reitsma et al. 2005). First, the main parameters to be estimated - global Se and global Sp - are analyzed jointly because they are negatively correlated (due to the threshold effect). Second, between-study variability is generally high in this type of meta-analysis. Thus, the use of hierarchical random effects models is necessary to appropriately take into account both within and between study variability. Third, the method employs a regression model that naturally allows the inclusion of study-level covariates to perform meta-regressions.

The main assumption of this hierarchical model is at the second level, namely that true values of the logit-transformed Ses and Sps of the individual studies should be independent and follow normal distributions (with unknown means and variances). The main parameters of interest that are estimated are the means of the underlying normal distributions of the Se and $\mathrm{Sp}$ values. It is important to use these normal distributions, as a priori the studies have different $\mathrm{Se}$ and $\mathrm{Sp}$ values due to numerous differences in the study characteristics. Logittransformed $\mathrm{Se}$ and $\mathrm{Sp}$ values are difficult to interpret directly. We therefore used the inverse logit transformation of the estimated means to obtain the global Se and Sp values.

Meta-regressions additionally assume homoscedasticity of heterogeneity variances, that is, between-study variances of the logit-sensitivity and logit-specificity parameters must remain approximately constant through the covariate levels. Here, the number of studies was too low to verify these assumptions. However, subgroup analyses were used to confirm meta-regression results without having to assume homoscedasticity. No zero-cell adjustment was needed since the model used binomial distributions to correctly model sampling uncertainty in both Se and $\mathrm{Sp}$.

As mentioned above, a category of cognition was metaanalyzed when it contained at least three studies. These meta-analyses all consisted of a small number of studies (from 3 to 6 ) with occasionally small sample sizes. For such metaanalyses, the maximum likelihood estimation of the bivariate and HSROC model parameters often suffer from convergence problems (Paul et al. 2010). Therefore, we chose the bivariate Bayesian implementation of the $\mathrm{R}$ package, meta4diag (Guo and Riebler 2016), which is based on the Integrated Nested Laplace Approximations method (Rue et al. 2009). 
The estimations of the variances and correlation of the joint distribution of Se and $\mathrm{Sp}$ values were not precise, because of the small number of studies, and were thus not informative. Since the model uses Bayesian inference, the reported credible intervals (CrI) for global sensitivities and specificities are credible intervals that correspond to the posterior distributions of the parameters. By definition, the estimated parameters lie within the CrIs with a posterior probability of $95 \%$ (Robert 2001). In other words, the CrIs must be interpreted to have a $95 \%$ certainty of containing the true parameter.

\section{Exploration of Sources of Heterogeneity}

We considered mean age and the length of follow-up of study participants to be potential sources of heterogeneity, as these variables could influence the pooled estimates. These analyses were performed through meta-regressions in which these variables were considered to be continuous covariates, one at a time. We also carried out subgroup analyses whenever the CrI of the effect of the covariate on Se or Sp was found to exclude the zero value. These analyses were performed by dividing the studies into two more or less equally numbered subgroups based on the covariate values.

\section{Sensitivity to Prior Distributions}

Vague prior distributions used in Bayesian analyses are generally expected to yield findings close to those obtained with other frequently used methods (Spiegelhalter et al. 1999). However, given the small number of studies and the sparse data involved in our meta-analyses, it was important to verify the sensitivity to prior distributions. The prior distributions that are susceptible to have an influence on the results are those of the heterogeneity variances and the correlation. However, as noted by Paul et al. (2010), the influence of the correlation prior is expected to be less for a data set with a lower correlation. Since we did not observe strong correlations in our data, we left the correlation prior as the default of the meta4diag function: normal distribution with a mean of 0 and a variance of 5 . Hence, our sensitivity analyses were performed by varying only the prior distribution of the heterogeneity variances. We took two of the priors suggested by (Paul et al. 2010), namely 1) mildly informative inverse gamma distribution with a shape of 0.25 and a rate of 0.025 and 2) the less informative inverse gamma distribution with both the shape and rate equal to 0.001 . The priors for the mean parameters and beta-coefficients of the covariates were also used as the default for both sensitivity and specificity, that is, normal prior with a mean of zero and a large variance (exact value of variance not specified by the authors of the package). The two priors for the heterogeneity variances usually yielded very close results, in which case only one was reported.

\section{Publication Bias}

The number of studies was insufficient to assess publication bias. Fortunately, this type of bias is less expected to occur for reviews of diagnostic test accuracy than for other types of reviews. In fact, Leeflang (2014) suggested that there is little evidence of publication bias for diagnostic test accuracy studies and hence publication bias is less of a concern for diagnostic test accuracy studies than for other types of reviews (Leeflang 2014).

\section{Subsidiary Analyses Excluding High Risk of Bias Studies}

Se and Sp values may be inflated by the inclusion of studies with a high risk of bias (Whiting et al. 2011). We performed subsidiary analyses after removal of all such studies when possible and then compared the results to those obtained when including all studies.

\section{Results}

\section{Study Selection}

The search strategy identified 4979 citations from electronic databases and six citations from hand searching. After adjusting for duplicates, 3355 citations remained in the pool of papers. After screening titles and abstracts, 313 citations were potentially eligible and full reports were retrieved and analyzed. We then excluded 285 studies which did not meet criteria based on a detailed examination of the full text, thus leaving 28 studies for the systematic review and meta-analysis. All were included in the systematic review. Of these, 21 met criteria described above to be included in the metaanalysis (Fig. 1).

\section{Study Characteristics}

The included studies and their characteristics are shown in Table 1. Among the 28 selected studies (references listed in Table 1), 22 were prospective, four were retrospective, and two used data from the Alzheimer's Disease Neuroimaging Initiative. The studies on which the review is based involved a total of 2365 participants with MCI. Participants were followed over a period of 12 to 60 months (mean follow-up: 31 $+/-14$ months). Among the enrolled MCI participants, 916 $(38.7 \%)$ later fulfilled criteria for a diagnosis of AD. The mean conversion rate varied from 6 to $39 \%$ per year based on studies that used a prospective study design.

Twenty-one of the 28 studies used Petersen's criteria to identify MCI at entry, consisting of subjective cognitive complaint, evidence for cognitive impairment (relative to age and education), preservation of general functional abilities, and the 
Fig. 1 PRISMA 2009 Flow Diagram

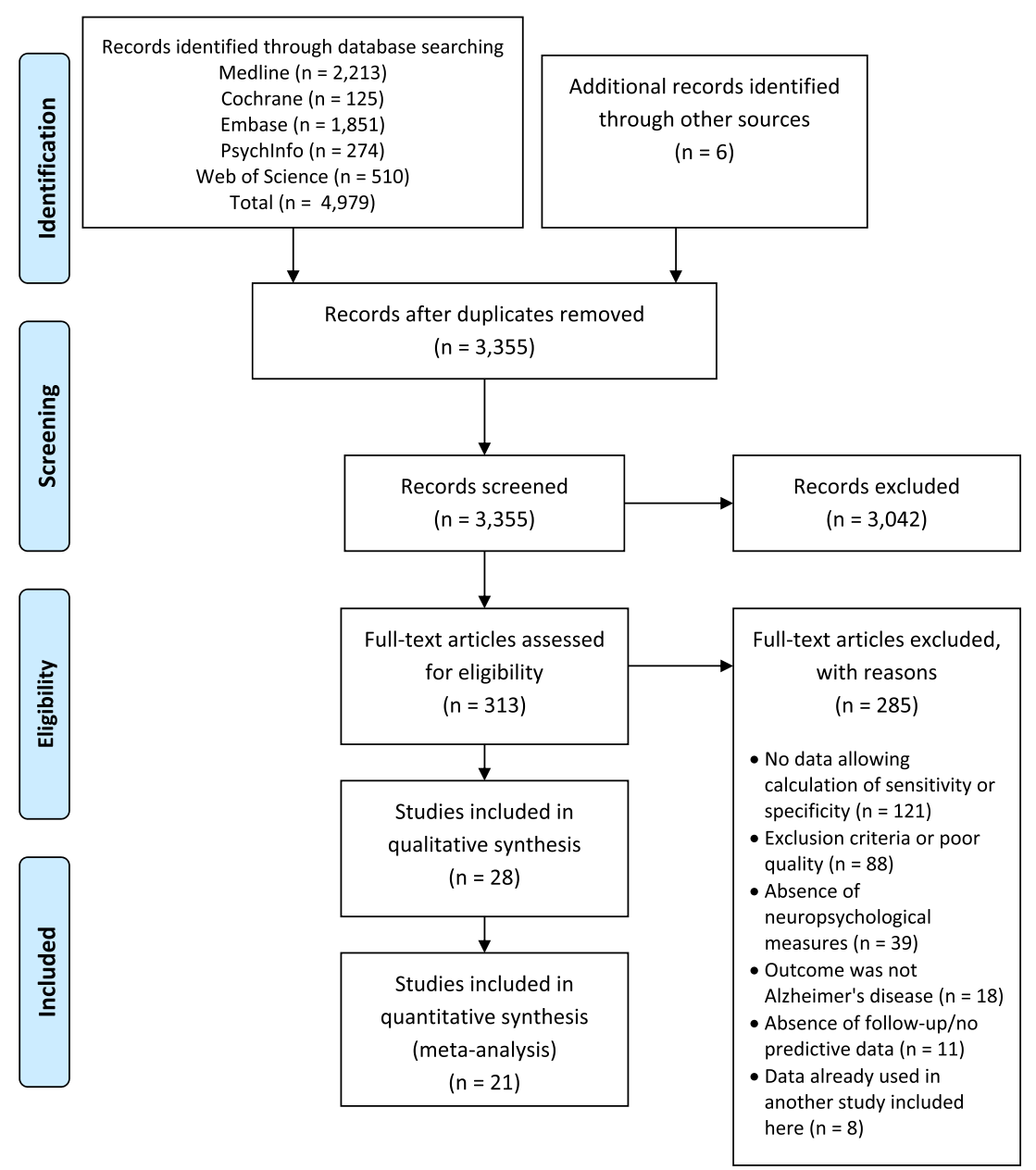

absence of diagnosed dementia. Among these 21 studies, 12 included both single and multiple domain amnestic MCI (aMCI), four included only single-domain amnestic MCI (sd-MCI), and five included amnestic and non-amnestic MCI. Four studies used the Global Deterioration Scale (GDS) to identify MCI (i.e., those with a score of 3 on the GDS). Three studies used other terminologies to describe their patients. Albert et al. (2001) selected participants with questionable Alzheimer's disease (QAD), defined as those with a score of 0.5 for the CDR (Albert et al. 2001). Galton et al. selected participants with "questionable dementia," defined by the presence of subjective complaints of memory impairment substantiated by an informant, (b) normal activities of daily living, and (c) absence of dementia as evident from an MMSE score of $\geq 23$, and a score of 0.5 on the CDR (Galton et al. 2005). Finally, Tierney et al., selected patients with a three-month history of symptomatic memory problems that interfered with daily functioning (GDS 2 or 3), no diagnosis of dementia, and an MMSE $\geq 24$ or a score $\geq 123$ on the Mattis Dementia Rating Scale (Mattis 1988; Tierney et al. 1996).

The mean age at entry for those who progressed to $\mathrm{AD}$ ranged from 64.9 to 77.6 years (mean age $=71.8$ years) versus 59.3 to 75 years (mean age $=69.8$ years) for non-progressors.
The percentage of females in the group of progressors varied from 33 to $77 \%$ (overall percentage $=54 \%$ ) vs 32 to $72 \%$ in the group of non-progressor MCI (overall percentage $=51 \%$ ). Mean years of education of participants who progressed to AD varied from 7.5 to 15.6 (mean $=12.1$ years) vs 7.7 to 15.9 (mean $=12.6$ years) for non-progressors. All included studies used the NINCDS-ARDRA criteria as their outcome for AD.

The studies reported predictive values for a total of 61 neuropsychological tests. Sixteen articles reported the predictive values of individual neuropsychological tests, five reported the predictive value of a combination of several neuropsychological tests, and seven included both individual tests and combinations of tests.

\section{Risk of Bias within Studies}

Table 2 shows the risk of bias associated with the included studies. Ten studies showed a low risk of bias, eight a moderate risk of bias, and ten a high risk of bias (all references listed in Table 2). The most frequent limitation comes from the selection process of the sample, which may not be representative of the population of interest. For example, Eckerstrom et al. selected, a posteriori, a sub-sample of non-progressors to match the 


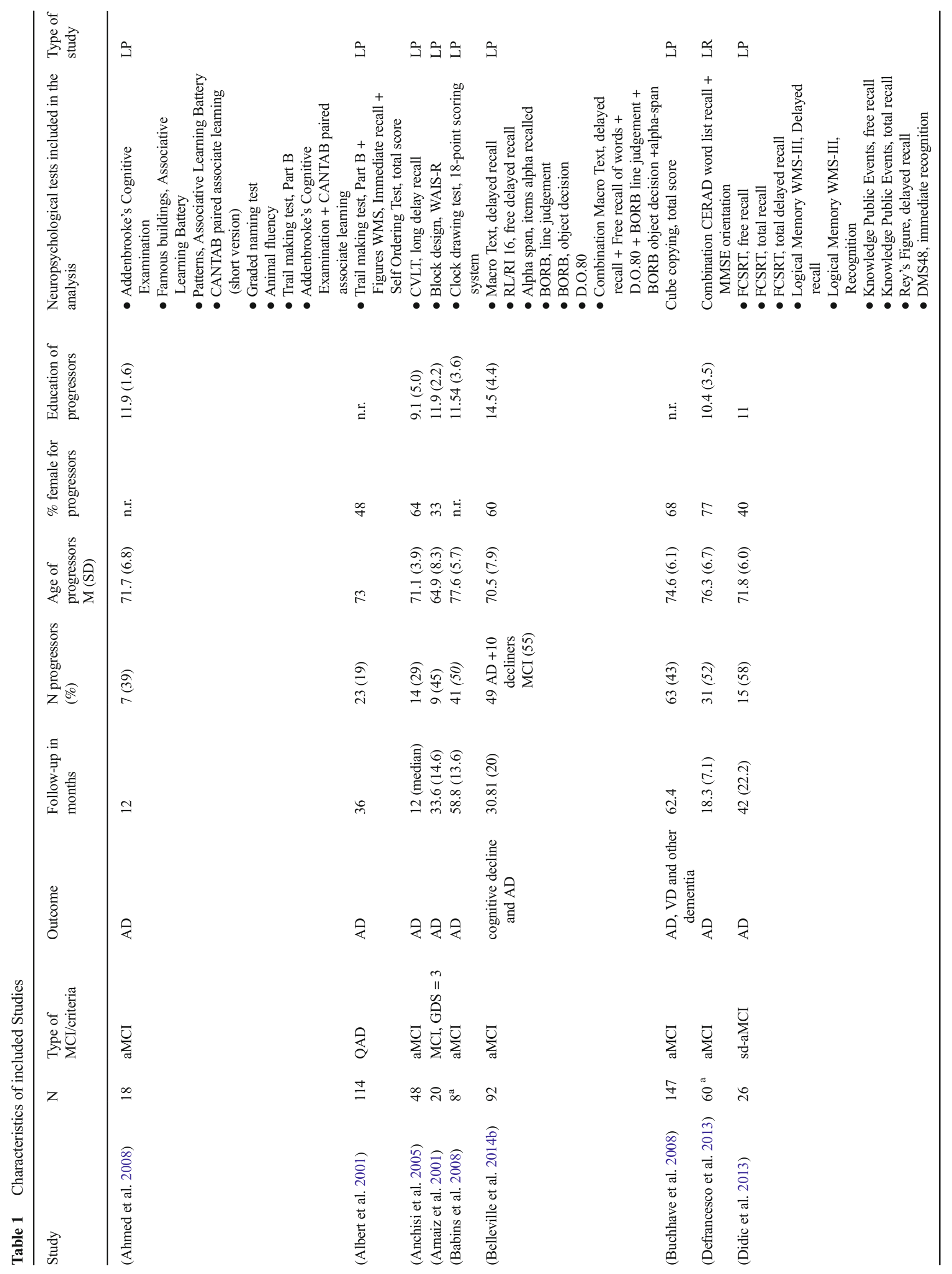




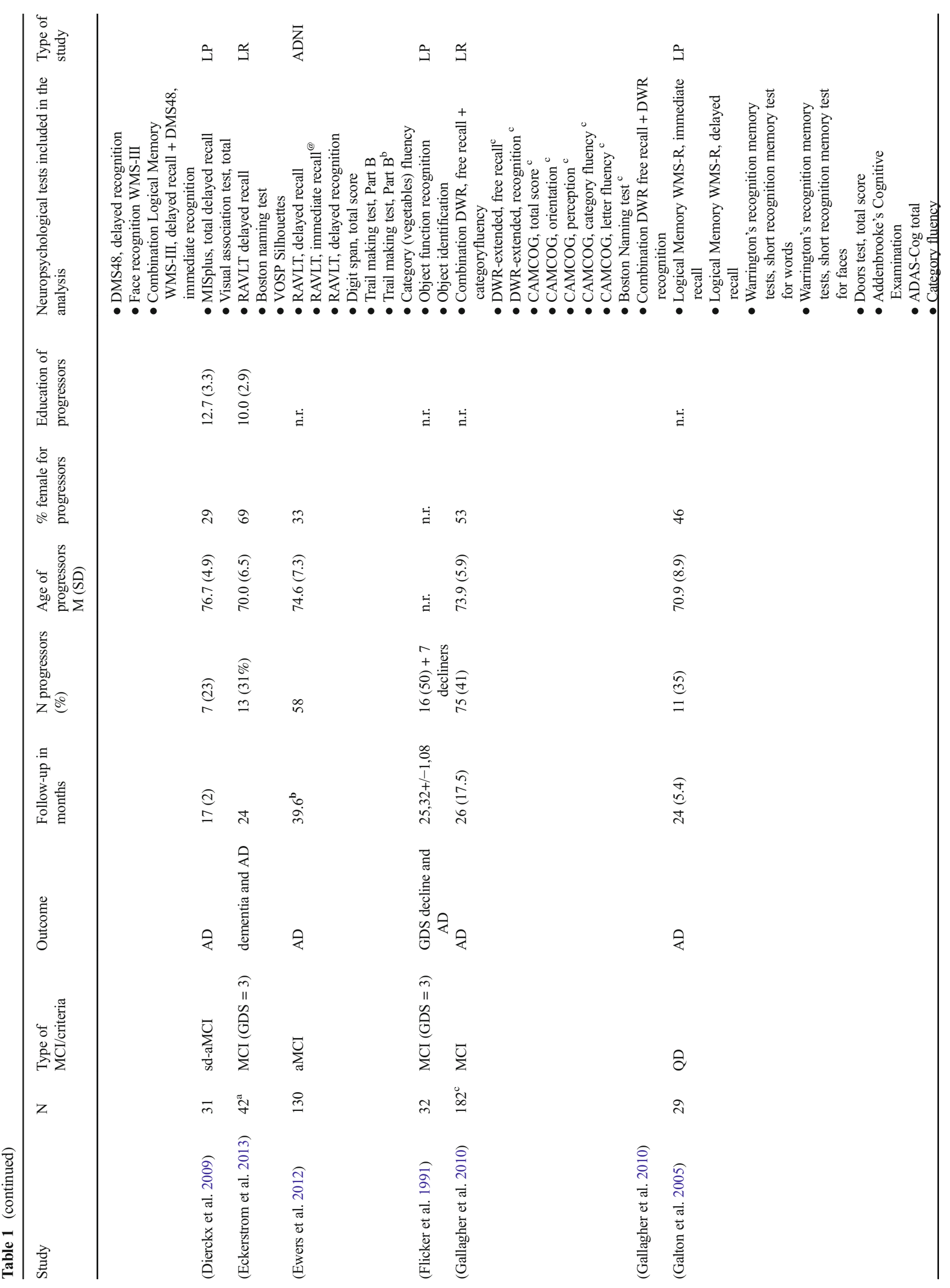




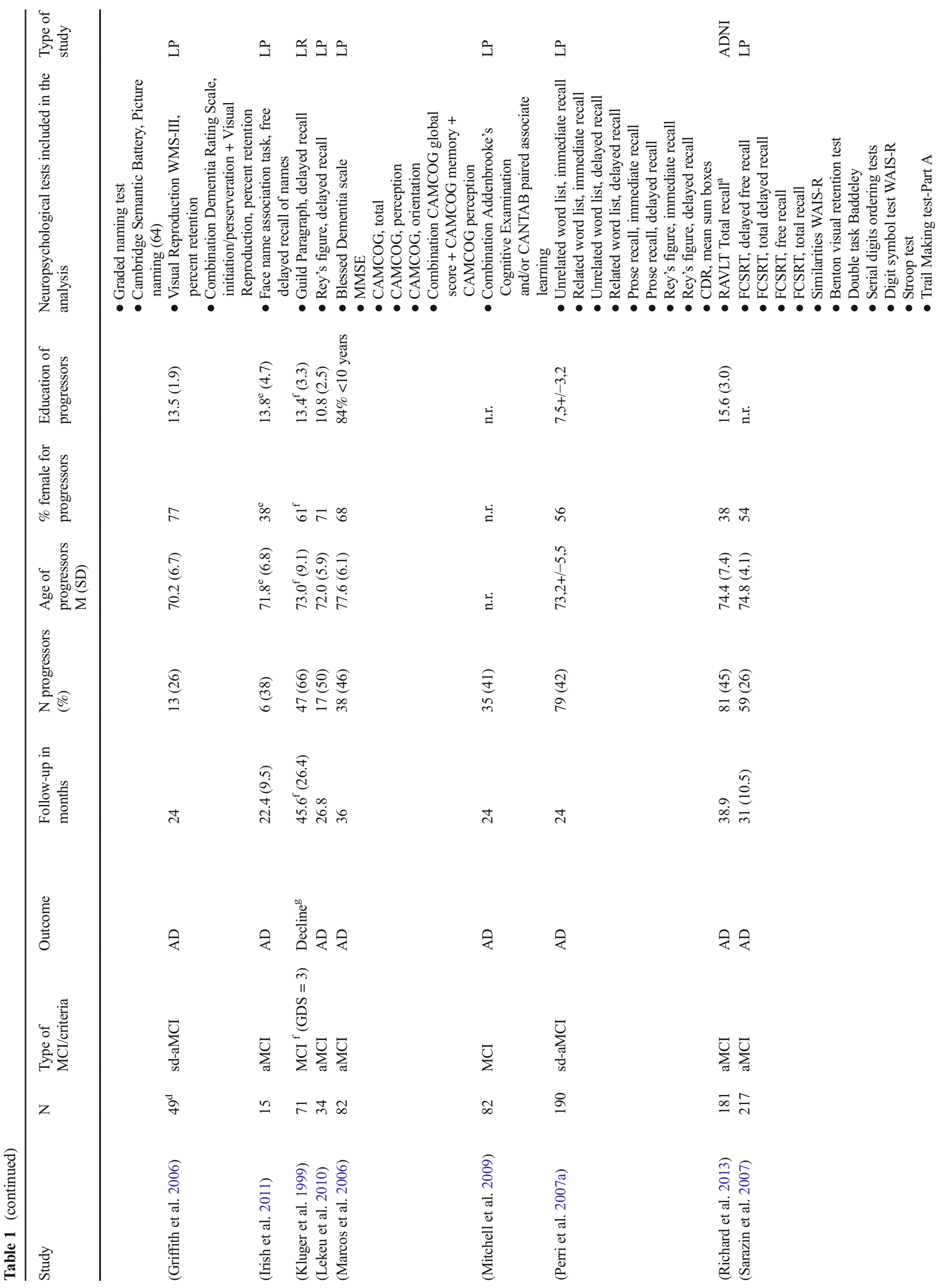




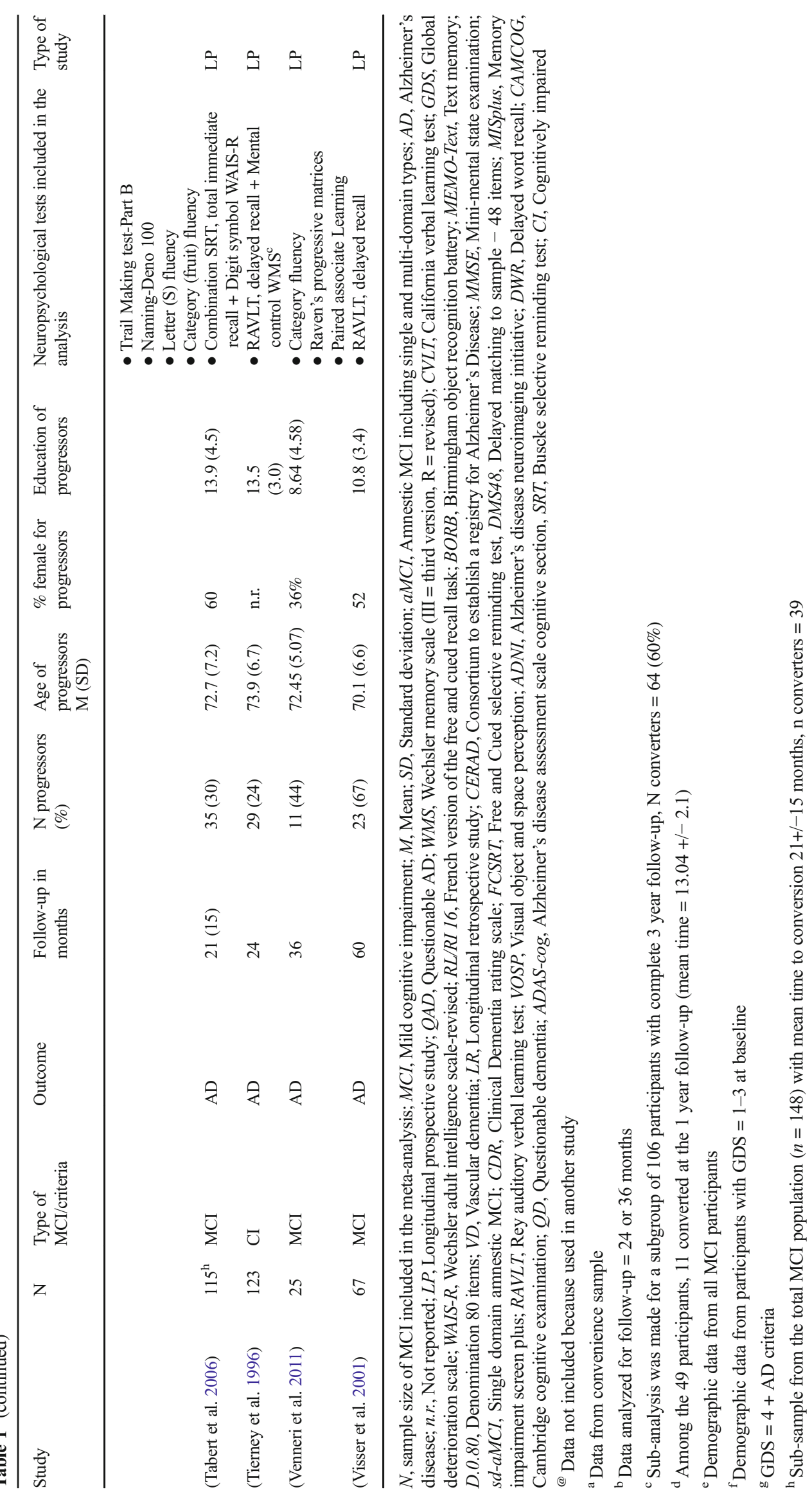




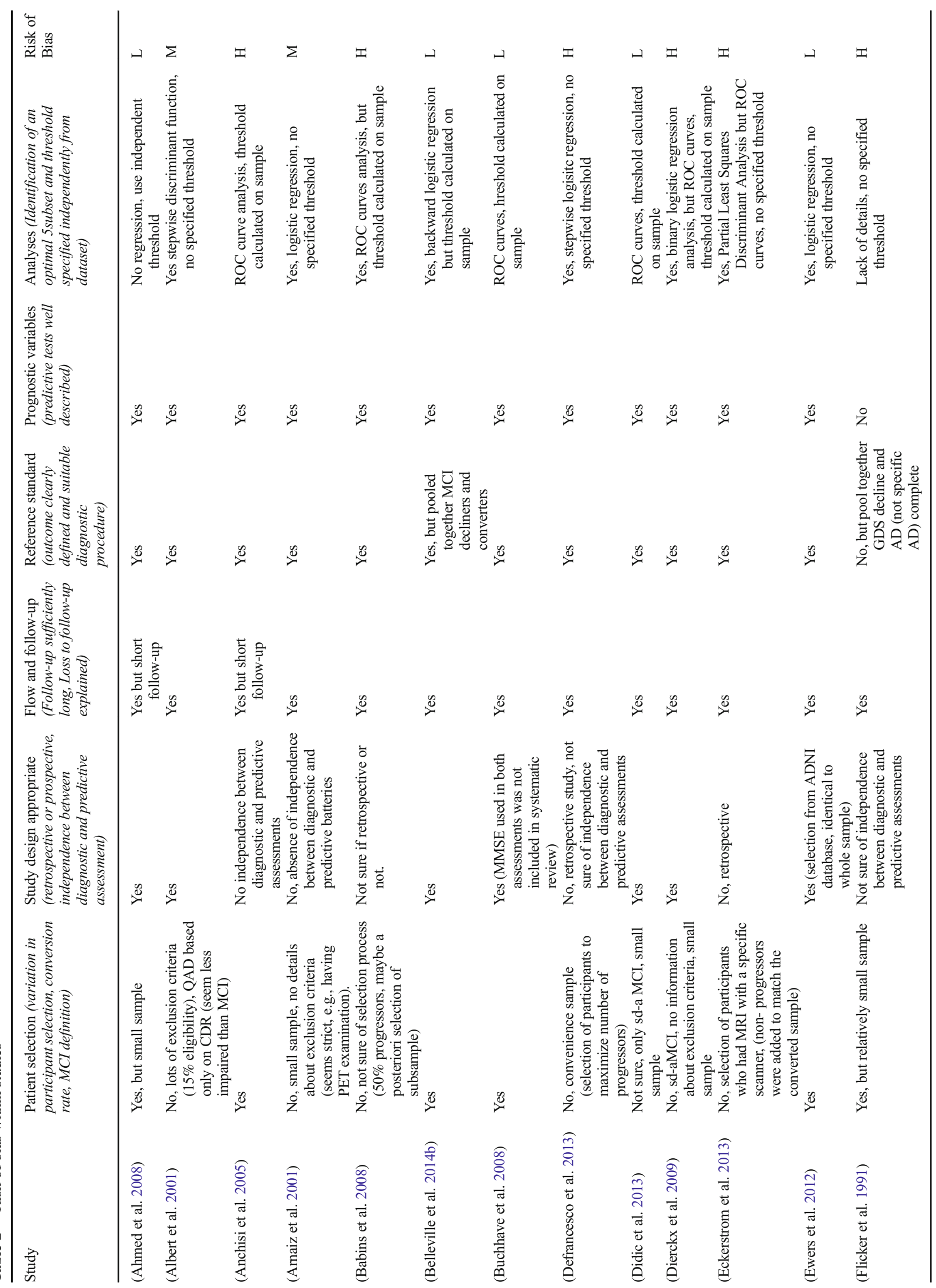




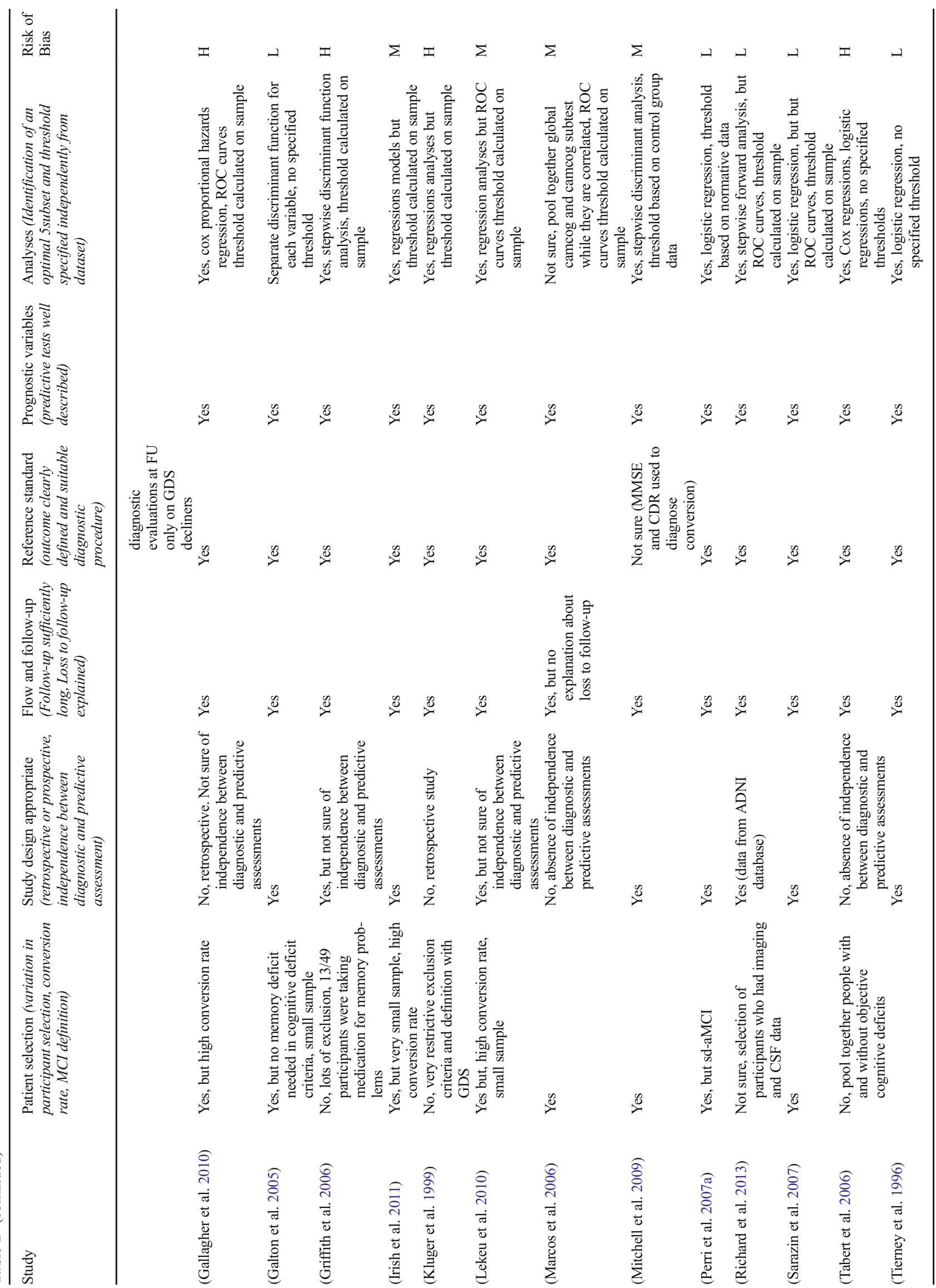


sample of progressors. Other studies only selected participants who previously underwent particular examinations (e.g., PET scan in Arnaiz et al. 2001 or a CSF examination in Richard et al. 2013). Griffith et al., included participants who were taking cholinesterase inhibitors (13 of $49 \mathrm{MCI}$ ). Other studies did not include sufficient details about the selection process (Babins et al. 2008). Many studies relied on a small sample size (<40 participants: Ahmed et al. 2008; Arnaiz et al. 2001; Didic et al. 2013; Dierckx et al. 2009; Flicker et al. 1991; Galton et al. 2005; Irish et al. 2011; Lekeu et al. 2010; Venneri et al. 2011) or showed a higher progression rate than expected (Gallagher et al. 2010; Irish et al. 2011; Lekeu et al. 2010).

Other aspects of the study design raised issues related to quality, albeit less frequently. For example, five studies did not ensure strict independence between the diagnostic battery and the predictive battery (Anchisi et al. 2005; Arnaiz et al. 2001; Marcos et al. 2006; Tabert et al. 2006; Venneri et al. 2011). Thus, either some of the neuropsychological tests included in the predictive battery were also used to make the baseline or follow-up diagnosis of the participants or the staff administering the diagnostic battery were not blind to the participants' performance on the predictive battery. Six studies did not provide sufficient information to determine whether the diagnostic and predictive assessments were independent with certainty (Defrancesco et al. 2013; Flicker et al. 1991; Gallagher et al. 2010; Griffith et al. 2006; Lekeu et al. 2010; Visser et al. 2001).

Most studies had a duration of follow-up longer than 12 months and most reported the reasons for loss to followup. Only two studies had a relatively short follow-up (12 months), which likely increased the risk of false negatives (Ahmed et al. 2008; Anchisi et al. 2005). Most studies used appropriate procedures for diagnosis. Two studies pooled patients who converted to $\mathrm{AD}$ and patients who showed significant cognitive decline (Belleville et al. 2014b; Flicker et al. 1991) and thus compared "stable MCI" to "decliners". Flicker et al. administrated the reference standard battery for AD diagnosis only to patients who showed decline on the GDS. Thus, this study presents a "partial bias verification". As per our inclusion criteria, all the predictive tests were well described or relied on tests that are well known and for which an extensive description is available from the literature.

Most studies used ROC curves to calculate optimal cut-offs for the tests and determine predictive accuracy. Twenty studies used regression analyses to assess and compare the predictive accuracy of different tests.

\section{Results from the Systematic Review}

A summary of the predictive accuracy values for single domains is presented in Table S3 (Supplementary material). The sensitivities observed from individual studies ranged from 0.18 to 1 and the specificities ranged from 0 to 1 . A very large 
Table 3 Individual tests with excellent specificity or sensitivity, grouped by cognitive domain

\begin{tabular}{|c|c|c|c|c|c|c|c|c|}
\hline & $\mathrm{TP}$ & FP & FN & $\mathrm{TN}$ & SE & SP & $\mathrm{ACC}$ & Cut-of \\
\hline \multicolumn{9}{|l|}{ Verbal episodic memory } \\
\hline \multicolumn{9}{|l|}{ Verbal immediate recall $* *$} \\
\hline Logical memory, immediate recall (Galton et al. 2005)* & 10 & 4 & 1 & 14 & 0.91 & 0.78 & 0.83 & n.r. \\
\hline FCSRT, free recall (Sarazin et al. 2007)*a & 42 & 13 & 17 & 145 & 0.71 & 0.92 & 0.86 & 17 \\
\hline \multicolumn{9}{|l|}{ World-list cued immediate recall with oriented encoding } \\
\hline FCSRT, total recall (Didic et al. 2013) & 10 & 1 & 5 & 10 & 0.67 & 0.91 & 0.77 & 37 \\
\hline FCSRT, total recall (Sarazin et al. 2007) ${ }^{\mathrm{a}}$ & 47 & 16 & 12 & 142 & 0.80 & 0.90 & 0.87 & 40 \\
\hline \multicolumn{9}{|l|}{ paragraph delayed recall $* *$} \\
\hline Logical memory, delayed recall (Galton et al. 2005)* & 10 & 4 & 1 & 14 & 0.91 & 0.78 & 0.83 & n.r. \\
\hline Logical memory, delayed recall (Didic et al. 2013)* & 11 & 0 & 4 & 11 & 0.73 & 1.00 & 0.85 & 6 \\
\hline Guild Paragraph, delayed recall (Kluger et al. 1999)* & 45 & 4 & 2 & 20 & 0.96 & 0.83 & 0.92 & n.r. \\
\hline \multicolumn{9}{|l|}{ Word-list free delayed recall with non oriented encoding** } \\
\hline RAVLT, delayed recall (Eckerstrom et al. 2013)* c & 12 & 1 & 1 & 20 & 0.92 & 0.94 & 0.94 & n.r. \\
\hline RAVLT, delayed recall (Visser et al. 2001)* & 17 & 3 & 6 & 41 & 0.74 & 0.93 & 0.87 & n.r. \\
\hline \multicolumn{9}{|l|}{ Word-list free delayed recall with oriented encoding** } \\
\hline CVLT, long delay free recall (Anchisi et al. 2005)* & 13 & 14 & 1 & 20 & 0.93 & 0.59 & 0.69 & 7 \\
\hline FCSRT, delayed free recall (Sarazin et al. 2007)* a & 45 & 15 & 14 & 143 & 0.76 & 0.91 & 0.87 & 6 \\
\hline Face-name association task, free delayed recall of names (Irish et al. 2011)* & 6 & 1 & 0 & 8 & 1.00 & 0.86 & 0.92 & 3 \\
\hline \multicolumn{9}{|l|}{ Word-list cued delayed recall with oriented encoding $* *$} \\
\hline FCSRT, total delayed recall (Didic et al. 2013)* & 9 & 1 & 6 & 10 & 0.60 & 0.91 & 0.73 & 12 \\
\hline MISplus total delayed recall free\&cued (Dierckx et al. 2009)* & 5 & 2 & 2 & 22 & 0.71 & 0.92 & 0.87 & 2 \\
\hline \multicolumn{9}{|l|}{ Paragraph recognition } \\
\hline Logical memory WMS-III recognition (Didic et al. 2013) & 5 & 0 & 6 & 9 & 0.45 & 1.00 & 0.70 & 18 \\
\hline \multicolumn{9}{|l|}{ Visual episodic memory } \\
\hline \multicolumn{9}{|l|}{ Immediate recall } \\
\hline Rey’s figure, immediate recall (Perri et al. 2007b) & 14 & 9 & 65 & 102 & 0.18 & 0.92 & 0.61 & n.r. \\
\hline \multicolumn{9}{|l|}{ Delayed recall** } \\
\hline Visual Reproduction, percent retention (Griffith et al. 2006)* & 10 & 3 & 3 & 33 & 0.77 & 0.91 & 0.88 & 26 \\
\hline \multicolumn{9}{|l|}{ Visual recognition } \\
\hline DSM48, immediate recognition (Didic et al. 2013) & 12 & 1 & 3 & 10 & 0.80 & 0.91 & 0.85 & 89 \\
\hline Face recognition WMS-III, immediate scaled score (Didic et al. 2013) & 14 & 6 & 1 & 5 & 0.93 & 0.45 & 0.73 & 12 \\
\hline \multicolumn{9}{|l|}{ Associative memory } \\
\hline Paired Associate Learning (Venneri et al. 2011) & 11 & 14 & 0 & 0 & 1.00 & 0.00 & 0.44 & n.r. \\
\hline CANTAB paired associate learning short version (Ahmed et al. 2008) & 7 & 5 & 0 & 6 & 1.00 & 0.55 & 0.72 & 14 \\
\hline Visual Association test total (Dierckx et al. 2009) & 3 & 1 & 4 & 23 & 0.43 & 0.96 & 0.84 & $1 / 6$ \\
\hline Patterns, Associative Learning Battery, errors (Ahmed et al. 2008) & 7 & 6 & 0 & 5 & 1.00 & 0.46 & 0.67 & 18 \\
\hline \multicolumn{9}{|l|}{ Language } \\
\hline \multicolumn{9}{|l|}{ Naming tests** } \\
\hline Boston naming test; 60 items (Eckerstrom et al. 2013)* c & 13 & 5 & 0 & 16 & 0.99 & 0.74 & 0.85 & n.r. \\
\hline Graded naming test (Ahmed et al. 2008)* & 3 & 1 & 4 & 10 & 0.40 & 0.91 & 0.72 & 14 \\
\hline \multicolumn{9}{|l|}{ Tests of semantic knowledge ${ }^{* *}$} \\
\hline Object function recognition (Flicker et al. 1991)* & 20 & 0 & 3 & 9 & 0.86 & 1.00 & 0.90 & n.r. \\
\hline Object identification (Flicker et al. 1991) & 13 & 0 & 10 & 9 & 0.57 & 1.00 & 0.69 & n.r. \\
\hline \multicolumn{9}{|l|}{ Semantic fluency** } \\
\hline Category fluency; animals, vegetables \& fruits (Gallagher et al. 2010)* & 60 & 22 & 4 & 20 & 0.94 & 0.48 & 0.75 & 36 \\
\hline Category fluency; animals (Ahmed et al. 2008)* & 2 & 0 & 5 & 11 & 0.29 & 1.00 & 0.72 & 11 \\
\hline Category fluency (Venneri et al. 2011)* & 10 & 7 & 1 & 7 & 0.91 & 0.50 & 0.68 & n.r. \\
\hline \multicolumn{9}{|l|}{ Visuo-constructive functions } \\
\hline \multicolumn{9}{|l|}{ Visuo-spatial tests** } \\
\hline VOSP Silhouettes (Eckerstrom et al. 2013)*c & 12 & 2 & 1 & 19 & 0.92 & 0.92 & 0.91 & n.r. \\
\hline
\end{tabular}


Table 3 (continued)

$\begin{array}{llllllll}\text { TP } & \text { FP } & \text { FN } & \text { TN } & \text { SE } & \text { SP } & \text { ACC } & \text { Cut-off }\end{array}$

Brief and Global measures

Global measures**

ACE Addenbrooke's Cognitive Examination (Ahmed et al. 2008)*

ACE Addenbrooke's Cognitive Examination (Galton et al. 2005)*

CAMCOG, total score (Marcos et al. 2006)*

$\begin{array}{llllllll}7 & 5 & 0 & 6 & 1.00 & 0.55 & 0.72 & 88 \\ 8 & 0 & 3 & 18 & 0.73 & 1.00 & 0.90 & \text { n.r. } \\ 35 & 14 & 3 & 30 & 0.92 & 0.68 & 0.79 & 79.5\end{array}$

$T P$, Number of true positive; $F P$, Number of false positive; $F N$, Number of false negative; $T N$, Number of true negative; $S E$, Sensitivity; $S P$, Specificity; $A C C$, Overall accuracy; n.r., Not reported

**Cognitive domains included in a meta-analysis

*data included in a meta-analysis

${ }^{\text {a }}$ Age corrected data

${ }^{\mathrm{b}}$ Age, gender and education corrected data

${ }^{\mathrm{c}}$ Age and education corrected data

number of tests show very good $(\geq 0.70)$ sensitivity, very good specificity, or both. The tests which obtained the sensitivity and specificity values in the excellent range $(\geq 0.90)$ are shown in Table 3. The table indicates predictive values (number of true positives, true negatives, false positives, false negatives, sensitivity, specificity, and accuracy) for each cognitive domain category. The cut-off values of the tests used in the studies are included, if available. Individual tests showed excellent specificity more often than excellent sensitivity. The tests with best overall accuracy $(\geq 0.90)$ are shown in Table 4 . Five individual tests and one global measure had excellent overall predictive value, implying that they combine very high

Table 4 Individual tests with excellent overall accuracy

\begin{tabular}{|c|c|c|c|c|c|c|c|c|}
\hline & $\mathrm{TP}$ & FP & FN & $\mathrm{TN}$ & SE & SP & $\mathrm{ACC}$ & Cut-off \\
\hline \multicolumn{9}{|l|}{ Verbal episodic memory } \\
\hline \multicolumn{9}{|l|}{ Paragraph delayed recall $* *$} \\
\hline Guild Paragraph, delayed recall (Kluger et al. 1999)* & 45 & 4 & 2 & 20 & 0.96 & 0.83 & 0.92 & n.r. \\
\hline \multicolumn{9}{|l|}{ Word-list free delayed recall with non oriented encoding $* *$} \\
\hline RAVLT, delayed Recall (Eckerstrom et al. 2013)* c & 12 & 1 & 1 & 20 & 0.92 & 0.94 & 0.94 & n.r. \\
\hline \multicolumn{9}{|l|}{ Word-list free delayed recall with oriented encoding** } \\
\hline Face-name association task, free delayed recall of names (Irish et al. 2011)* & 6 & 1 & 0 & 8 & 1 & 0.86 & 0.92 & 3 \\
\hline \multicolumn{9}{|l|}{ Language } \\
\hline \multicolumn{9}{|l|}{ Tests of semantic knowledge** } \\
\hline Object function recognition (Flicker et al. 1991)* & 20 & 0 & 3 & 9 & 0.86 & 1.00 & 0.90 & n.r. \\
\hline \multicolumn{9}{|l|}{ Visuo-constructive functions } \\
\hline \multicolumn{9}{|l|}{ Visuo-spatial tests** } \\
\hline VOSP Silhouettes (Eckerstrom et al. 2013)*c & 12 & 2 & 1 & 19 & 0.92 & 0.92 & 0.91 & n.r. \\
\hline \multicolumn{9}{|l|}{ Brief and Global measures } \\
\hline \multicolumn{9}{|l|}{ Global measures** } \\
\hline ACE Addenbrooke's Cognitive Examination (Galton et al. 2005)* & 8 & 0 & 3 & 18 & 0.73 & 1.00 & 0.90 & n.r. \\
\hline \multicolumn{9}{|c|}{$\begin{array}{l}T P \text {, Number of true positive; } F P \text {, Number of false positive; } F N \text {, Number of false negative; } T N \text {, Number of true negative; } S E \text {, Sensitivity; } S P \text {, Specificity; } \\
A C C \text {, Overall accuracy; } n . r ., \text { Not reported }\end{array}$} \\
\hline \multicolumn{9}{|l|}{ *Data included in a meta-analysis } \\
\hline \multicolumn{9}{|l|}{$* *$ Cognitive domains included in a meta-analysis } \\
\hline \multicolumn{9}{|l|}{${ }^{\mathrm{a}}$ Age corrected data } \\
\hline${ }^{\mathrm{b}}$ Age, gender and education corrected data & & & & & & & & \\
\hline${ }^{\mathrm{c}}$ Age and education corrected data & & & & & & & & \\
\hline
\end{tabular}


specificity and sensitivity. The tests were 1) the Guild Paragraph, delayed recall, 2) the RAVLT, delayed Recall, 3) Face-name association task, free delayed recall of names, 4) Object function recognition, 5) VOSP Silhouettes, and the ACE Addenbrooke's Cognitive Examination.

Results obtained from combining different neuropsychological tests are provided in Table 4 (Supplementary material). Most studies combined episodic memory tests with global measures or non-memory measures. Studies that examined a combination of memory and non-memory tests obtained very high to excellent predictive accuracy, with a few exceptions. Combining episodic memory tests with executive function tests gave very good sensitivities (from 0.74 to 0.77 ) and excellent specificities (0.83 to 0.94: Albert et al. 2001; Griffith et al. 2006; Tabert et al. 2006; Tierney et al. 1996). The combination of episodic memory with category fluency allowed for excellent sensitivity (0.84) and very good specificity (0.79: Gallagher et al. 2010). One study reported high sensitivity and specificity ( 0.88 and 0.87 , respectively) when combining episodic memory, language, visual perception, and executive function tasks (Belleville et al. 2014b).

Two studies combining an associative memory task (CANTAB Paired associate learning) with a global measure (Addenbrooke's Cognitive Examination) reported different results. Ahmed et al. reported excellent accuracy ( 1 and 0.82 for sensitivity and specificity, respectively), whereas Mitchell et al. reported high sensitivity (0.94), but low specificity $(0.40)$. This may be due to the difference in the duration of follow-up (12 versus 24 months) or study sample (18 versus 82 patients). One study reported that combining associative memory tests with language (category fluency) or visuo-constructive tasks provided very high sensitivities $(0.73$ to 0.91$)$, but low specificities $(0.5$ to 0.57 : Venneri et al. 2011). The same study reported low sensitivity and specificity values ( 0.64 and 0.5 , respectively) for a combination of associative memory, language measures, and visuoconstructive tasks (Venneri et al. 2011). However, this study was carried out with a small sample size $(n=25)$.

Combining episodic memory with global measures generally provided very good to excellent predictive accuracy. The combination of an episodic memory test with a brief measure (MMSE orientation) provided very good sensitivity and excellent specificity (0.74 and 0.9, respectively: Defrancesco et al. 2013). Combining episodic memory with global measures and visual perception showed excellent sensitivity (0.92) and very good specificity (0.73: Marcos et al. 2006). One study that combined episodic memory tests with a global measure (CDR mean sum of boxes) obtained low sensitivity ( 0.66$)$, but high specificity $(0.86$ : Perri et al. 2007b). The combination of different episodic memory modalities gave variable results ranging from low sensitivity (0.44) and high specificity (0.9: Gallagher et al. 2010) to excellent sensitivity and specificity (1: Didic et al. 2013).

Given the large variability observed in the predictive accuracy obtained from individual tests, the meta-analysis presented below, summarizes predictive accuracy values (sensitivity and specificity) by domain subgroups. We then present the results from meta-regression, which examined the interaction of sensitivity and specificity with the duration of followup, as a proxy for the time to diagnosis, and age.

\section{Results of the Meta-Analyses}

Convergence was achieved in all meta-analyses, except one (associative memory), and only two meta-regressions (verbal immediate recall and paragraph delayed recall) had one prior distribution for which convergence failed. In addition, the second prior distribution analysis failed for short follow-up subgroup for semantic fluency.

Verbal Episodic Memory Most tests measuring verbal episodic memory reported very good predictive accuracy (all above 0.7 ) and there was little variability related to the test condition (e.g., delayed or immediate, with or without oriented encoding, with or without cues at recall) or the particular material (e.g., words or paragraph). The only exceptions were for recognition and word-list cued delayed recall with oriented encoding, which had lower sensitivity values than other verbal memory tests. Predictive accuracy for tests of verbal immediate recall was reported in five studies (Galton et al. 2005; Perri et al. 2007b; Didic et al. 2013; Sarazin et al. 2007; Richard et al. 2013). The combined value was 0.745 for sensitivity (95\% CrI: $0.511-0.905)$ and 0.771 for specificity (95\% CrI: 0.581-0.897). Predictive accuracy for paragraph delayed recall was reported in five studies (Didic et al. 2013; Galton et al. 2005; Belleville et al. 2014b; Kluger et al. 1999; Perri et al. 2007b). The combined analysis gave a 0.776 sensitivity (95\% CrI: $0.541-0.928)$ and a 0.794 specificity (95\% CrI: 0.517-0.961). Word-list free delayed recall with nonoriented encoding was reported in four studies (Eckerstrom et al. 2013; Ewers et al. 2012; Visser et al. 2001; Perri et al. 2007b). The combined analysis gave 0.742 sensitivity (95\% CrI: $0.625-0.868)$ and a 0.814 specificity (95\% CrI: 0.517 0.961 ). Word-list free delayed recall with oriented encoding was also reported in five studies (Anchisi et al. 2005; Gallagher et al. 2010; Sarazin et al. 2007; Belleville et al. 2014b; Irish et al. 2011). The combined analysis gave 0.781 sensitivity (95\% CrI: $0.704-0.867$ ) and a 0.797 specificity (95\% CrI: 0.651-0.893). Word-list cued delayed recall with oriented encoding was reported in three studies (Sarazin et al. 2007; Didic et al. 2013; Dierckx et al. 2009). The combined analysis gave 0.676 sensitivity (95\% CrI: $0.526-0.796$ ) and 0.896 specificity (95\% CrI: $0.822-0.947$ ). Word-list recognition was reported in three studies (Galton et al. 2005; Ewers et al. 2012; Gallagher et al. 2010). The combined analysis gave 0.547 sensitivity (95\% CrI: $0.428-0.671)$ and 0.789 specificity (95\% CrI: 0.602-0.927). 
Visual Episodic Memory Four studies reported predictive accuracy for visual episodic memory tests (Didic et al. 2013; Griffith et al. 2006; Lekeu et al. 2010; Perri et al. 2007b). The combined analysis showed a relatively low sensitivity, 0.676 (95\% CrI: $0.413-0.889$ ), but very good specificity, 0.847 (95\% CrI: 0.722-0.913).

Language The predictive accuracy provided by language tests was relatively high with values just below 0.7 for sensitivity and somewhat higher for specificity. Predictive accuracy for naming tests was reported in six studies (Eckerstrom et al.
2013; Gallagher et al. 2010; Ahmed et al. 2008; Galton et al. 2005; Belleville et al. 2014b; Sarazin et al. 2007). The analysis gave a combined sensitivity score of 0.699 (95\% CrI: $0.51-$ $0.868)$ and a combined specificity score of 0.707 (95\% CrI: 0.621-0.813). Data for tests of semantic knowledge was reported in three studies (Didic et al. 2013; Sarazin et al. 2007; Flicker et al. 1991). The analysis gave a combined sensitivity score of 0.703 (95\% CrI: 0.442-0.906) and a higher combined specificity of 0.814 (95\% CrI: 0.631-0.984). Tests of semantic fluency were examined in six studies (Gallagher et al. 2010; Galton et al. 2005; Ahmed et al. 2008; Ewers et al. 2012;

Table 5 Meta-analyses

\begin{tabular}{|c|c|c|c|c|c|c|}
\hline \multirow[t]{2}{*}{ Cognitive domains } & \multicolumn{3}{|c|}{ All studies } & \multicolumn{3}{|c|}{ High risk of bias studies excluded } \\
\hline & $\mathrm{N}$ & Sensitivity $[\mathrm{CrI}]$ & Specificity $[\mathrm{CrI}]$ & $\mathrm{N}$ & Sensitivity $[\mathrm{CrI}]$ & Specificity $[\mathrm{CrI}]$ \\
\hline \multicolumn{7}{|l|}{ Verbal episodic memory } \\
\hline Verbal immediate recall & 5 & $\begin{array}{l}0.745^{\mathrm{a}} \\
{[0.511-0.905]}\end{array}$ & $\begin{array}{l}0.771^{\mathrm{a}} \\
{[0.581-0.897]}\end{array}$ & \multicolumn{3}{|c|}{ No high risk of bias study } \\
\hline Paragraph delayed recall & 5 & $\begin{array}{l}0.776 \\
{[0.541-0.928]}\end{array}$ & $\begin{array}{l}0.794 \\
{[0.675-0.914]}\end{array}$ & 4 & $\begin{array}{l}0.636 \\
{[0.510-0.808]}\end{array}$ & $\begin{array}{l}0.795 \\
{[0.623-0.953]}\end{array}$ \\
\hline $\begin{array}{l}\text { Word-list free delayed recall } \\
\text { with non oriented encoding, }\end{array}$ & 4 & $\begin{array}{l}0.742 \\
{[0.625-0.868]}\end{array}$ & $\begin{array}{l}0.814 \\
{[0.517-0.961]}\end{array}$ & 3 & $\begin{array}{l}0.707 \\
{[0.584-0.815]}\end{array}$ & $\begin{array}{l}0.744 \\
{[0.354-0.950]}\end{array}$ \\
\hline $\begin{array}{l}\text { Word-list free delayed recall } \\
\text { with oriented encoding }\end{array}$ & 5 & $\begin{array}{l}0.781 \\
{[0.704-0.867]}\end{array}$ & $\begin{array}{l}0.797 \\
{[0.651-0.893]}\end{array}$ & 3 & $\begin{array}{l}0.765 \\
{[0.647-0.891]}\end{array}$ & $\begin{array}{l}0.869 \\
{[0.717-0.944]}\end{array}$ \\
\hline $\begin{array}{l}\text { Word-list cued delayed recall } \\
\text { with oriented encoding }\end{array}$ & 3 & $\begin{array}{l}0.676 \\
{[0.526-0.796]}\end{array}$ & $\begin{array}{l}0.896 \\
{[0.822-0.947]}\end{array}$ & 2 & Not analyzable & \\
\hline Word-list recognition & 3 & $\begin{array}{l}0.547 \\
{[428-0.671]}\end{array}$ & $\begin{array}{l}0.789 \\
{[0.602-0.927]}\end{array}$ & 2 & Not analyzable & \\
\hline \multicolumn{7}{|l|}{ Visual episodic memory } \\
\hline Delayed recall & 4 & $\begin{array}{l}0.676 \\
{[0.413-0.889]}\end{array}$ & $\begin{array}{l}0.847 \\
{[0.722-0.913]}\end{array}$ & 3 & $\begin{array}{l}0.650 \\
{[0.320-0.912]}\end{array}$ & $\begin{array}{l}0.814 \\
{[0.624-0.904]}\end{array}$ \\
\hline \multicolumn{7}{|l|}{ Language } \\
\hline Naming tests & 6 & $\begin{array}{l}0.699 \\
{[0.51-0.868]}\end{array}$ & $\begin{array}{l}0.707 \\
{[0.621-0.813]}\end{array}$ & 4 & $\begin{array}{l}0.571 \\
{[0.458-0.683]}\end{array}$ & $\begin{array}{l}0.742 \\
{[0.622-0.887]}\end{array}$ \\
\hline Tests of semantic knowledge & 3 & $\begin{array}{l}0.703^{\mathrm{b}} \\
{[0.442-0.906]}\end{array}$ & $\begin{array}{l}0.814^{\mathrm{b}} \\
{[0.631-0.984]}\end{array}$ & 2 & Not analyzable & \\
\hline Semantic fluency & 6 & $\begin{array}{l}0.708 \\
{[0.468-0.875]}\end{array}$ & $\begin{array}{l}0.7 \\
{[0.539-0.836]}\end{array}$ & 5 & $\begin{array}{l}0.598 \\
{[0.425-0.779]}\end{array}$ & $\begin{array}{l}0.744 \\
{[0.565-0.891]}\end{array}$ \\
\hline \multicolumn{7}{|l|}{ Executive functions } \\
\hline Switching tests & 3 & $\begin{array}{l}0.541 \\
{[0.314-0.701]}\end{array}$ & $\begin{array}{l}0.679 \\
{[0.542-0.797]}\end{array}$ & \multicolumn{3}{|c|}{ No high risk of bias study } \\
\hline Working memory tests & 3 & $\begin{array}{l}0.599 \\
{[0.463-0.724]}\end{array}$ & $\begin{array}{l}0.667 \\
{[0.573-0.747]}\end{array}$ & \multicolumn{3}{|c|}{ No high risk of bias study } \\
\hline \multicolumn{7}{|l|}{ Visuo-constructive functions } \\
\hline Visuo-spatial tests & 4 & $\begin{array}{l}0.68 \\
{[0.366-0.914]}\end{array}$ & $\begin{array}{l}0.749 \\
{[0.613-0.873]}\end{array}$ & 2 & Not analyzable & \\
\hline Visuo-constructive tasks & 4 & $\begin{array}{l}0.637 \\
{[0.471-0.778]}\end{array}$ & $\begin{array}{l}0.643 \\
{[0.496-0.775]}\end{array}$ & 3 & $\begin{array}{l}0.561 \\
{[0.399-0.722]}\end{array}$ & $\begin{array}{l}0.705 \\
{[0.557-0.823]}\end{array}$ \\
\hline Brief/global measures & 4 & $\begin{array}{l}0.852^{\mathrm{c}} \\
{[0.73-0.946]}\end{array}$ & $\begin{array}{l}0.757^{\mathrm{c}} \\
{[0.468-0.966]}\end{array}$ & 3 & $\begin{array}{l}0.899 \\
{[0.721-0.981]}\end{array}$ & $\begin{array}{l}0.851 \\
{[0.294-1.00]}\end{array}$ \\
\hline
\end{tabular}

Global estimate (median of posterior distribution) and $95 \%$ credible intervals (CrI) of sensitivity and specificity of tests assessing cognitive domains. N indicates the number of study results included in each category

${ }^{\mathrm{a}}$ For these data, an alternative prior distribution gave the following results: Sensitivity $=0.747$ CrI [0.49-0.915], Specificity $=0.772$ [0.564-0.904]

${ }^{\mathrm{b}}$ For these data, an alternative prior distribution gave the following results: Sensitivity $=0.708$ CrI [0.385-0.927], Specificity $=0.834$ [0.478-1]

${ }^{\mathrm{c}}$ For these data, an alternative prior distribution gave the following results: Sensitivity $=0.853 \mathrm{CrI}[0.705-0.923]$, Specificity $=0.769$ [0.38-0.987] 
Sarazin et al. 2007; Venneri et al. 2011). The combined sensitivity score was 0.708 (95\% CrI: $0.468-0.875)$ and the combined specificity score 0.7 (95\% CrI: $0.539-0.836$ ).

Executive Functions This domain yielded relatively low predictive accuracy values with better specificity than sensitivity. Three studies reported predictive accuracy for switching tests (Trail test; Ahmed et al. 2008; Sarazin et al. 2007; Ewers et al. 2012). The analysis gave a combined sensitivity score of 0.541 (95\% CrI: 0.314-0.701) and a somewhat higher combined specificity score of 0.679 (95\% CrI: $0.542-0.797$ ). Three studies reported predictive accuracy for working memory tests (Ewers et al. 2012; Sarazin et al. 2007; Belleville et al. 2014b). The combined sensitivity score was 0.599 (95\% CrI: $0.463-0.724$ ) and the specificity was again slightly better with a combined score of 0.679 (95\% CrI: 0.573-0.747)

Visuo-Constructive Functions Four studies reported predictive accuracy for visuo-spatial tests (Gallagher et al. 2010; Marcos et al. 2006; Belleville et al. 2014b; Eckerstrom et al. 2013). The combined sensitivity score was 0.68 (95\% CrI: $0.366-0.914)$ and the combined specificity score was 0.749 (95\% CrI: 0.613-0.873). Three studies reported data from visuo-constructive tasks (Babins et al. 2008; Buchhave et al. 2008; Arnaiz et al. 2001). Their combined value was 0.637 (95\% CrI: 0.471-0.778) for sensitivity and 0.643 (95\% CrI: $0.496-0.775)$ for specificity.

Brief or Global Measures Four studies reported predictive accuracy for brief or global cognitive measures and their combined predictive accuracy was quite high, both in terms of sensitivity and specificity (Ahmed et al. 2008; Galton et al. 2005; Gallagher et al. 2010; Marcos et al. 2006). The analysis gave a combined sensitivity score of 0.852 (95\% CrI: $0.73-$ $0.946)$ and a combined specificity score of 0.757 (95\% CrI: 0.468-0.966).
Analyses Excluding the High Risk of Bias Studies The results of the meta-analyses performed after removal of the 10 studies with a high risk of bias are presented on the right-hand side of Table 5. Three test categories did not require reanalysis as they did not contain any high risk of bias studies (verbal immediate recall, switching tests, and working memory tests). Specificity and sensitivity scores obtained when excluding high-risk studies generally remained close to the values obtained from the initial analyses. In three cases, sensitivities that were above 0.70 fell below that score when excluding high-risk studies: paragraph delayed recall, naming, and semantic fluency. However, for naming and semantic fluency, lower sensitivities were partially compensated by slightly higher specificities. In the case of the visuo-constructive tasks category, the specificity increased from below 0.70 to very good (above 0.70 ) when excluding high-risk studies. Four test categories could not be re-analyzed because they ended up with only two studies: word-list cued delayed recall with oriented encoding, word-list recognition, semantic knowledge and visuo-spatial tests. No sensitivity analyses were performed for the subgroups as they all contained only three studies.

\section{Results of the Meta-Regression}

The results of the meta-regressions are presented in Table 6 . There was a negative association between the length of follow-up and specificity values for the naming tests (-0.094, 95\% CrI: $-0.173--0.027$ : Eckerstrom et al. 2013; Gallagher et al. 2010; Ahmed et al. 2008; Galton et al. 2005; Belleville et al. 2014b; Sarazin et al. 2007). Shorter mean follow-up was associated with higher specificity values. When examining specificity in the length of follow-up subgroups (Table 7), the combined specificity value for the naming tests obtained from the three studies with the longest follow-up (31 to 36 months: Gallagher et al. 2010; Belleville et al. 2014b; Sarazin et al. 2007) was good (0.648; 95\% CrI:

Table 6 Meta-regressions assessing the effect of follow-up duration or age of participants at baseline on sensitivity and specificity of neuropsychological tests

\begin{tabular}{|c|c|c|c|c|}
\hline \multirow[t]{2}{*}{ Cognitive domains } & \multicolumn{2}{|l|}{ Follow-up effect } & \multicolumn{2}{|l|}{ Age effect } \\
\hline & Effect on logit(Se) $[\mathrm{CrI}]$ & Effect on logit(Sp) [CrI] & Effect on logit(Se) $[\mathrm{CrI}]$ & Effect on $\operatorname{logit}(\mathrm{Sp})[\mathrm{CrI}]$ \\
\hline Paragraph delayed recall & $\begin{array}{l}0.071 \\
{[-0.123,0.245]}\end{array}$ & $\begin{array}{l}0.070 \\
{[-0.032,0.205]}\end{array}$ & $\begin{array}{l}0.145 \\
{[-0.567,0.791]}\end{array}$ & $\begin{array}{l}-0.243 \\
{[-0.688,0.399]}\end{array}$ \\
\hline $\begin{array}{l}\text { Word-list, free delayed recall } \\
\text { with oriented encoding }\end{array}$ & $\begin{array}{l}-0.058 \\
{[-0.165,0.014]}\end{array}$ & $\begin{array}{l}0.046 \\
{[-0.073,0.151]}\end{array}$ & NA & NA \\
\hline Naming & $\begin{array}{l}0.026 \\
{[-0.184,0.229]}\end{array}$ & $\begin{array}{l}-0.094 \\
{[-0.173,-0.027]}\end{array}$ & $\begin{array}{l}-0.129 \\
{[-0.658,0.354]}\end{array}$ & $\begin{array}{l}-0.114 \\
{[-0.399,0.148]}\end{array}$ \\
\hline Semantic fluency & $\begin{array}{l}0.143 \\
{[0.035,0.261]}\end{array}$ & $\begin{array}{l}-0.092 \\
{[-0.242,0.003]}\end{array}$ & $\begin{array}{l}-0.011 \\
{[-0.571,0.560]}\end{array}$ & $\begin{array}{l}-0.064 \\
{[-0.570,0.391]}\end{array}$ \\
\hline
\end{tabular}

Meta-regression results for the four cognitive domains containing five or more studies. Follow-up length and mean age were analyzed separately and both effects on Se and $\mathrm{Sp}$ (on the logit scale) were assessed 
Table 7 Meta-analyses of subgroups of studies with long or short follow-up

\begin{tabular}{|c|c|c|c|c|}
\hline & $\mathrm{N}$ & Sensitivity $[\mathrm{CrI}]$ & Specificity $[\mathrm{CrI}]$ & $\begin{array}{l}\text { Follow-up } \\
\text { (months) }\end{array}$ \\
\hline Naming (short FU) & 3 & $0.842^{\mathrm{a}}$ & $0.852^{\mathrm{a}}$ & $12-24$ \\
\hline Naming (long FU) & 3 & $\begin{array}{l}{[0.256,1]} \\
0.653\end{array}$ & $\begin{array}{l}{[0.703,0.943]} \\
0.648\end{array}$ & $31-36$ \\
\hline Semantic fluency (short FU) & 3 & $\begin{array}{l}{[0.437,0.825]} \\
0.540^{\mathrm{a}}\end{array}$ & $\begin{array}{l}{[0.539,0.738]} \\
0.765^{\mathrm{a}}\end{array}$ & $12-24$ \\
\hline & & {$[0.324,0.722]$} & {$[0.437,0.998]$} & \\
\hline Semantic fluency (long FU) & 3 & $\begin{array}{l}0.842 \\
{[0.569,0.966]}\end{array}$ & $\begin{array}{l}0.640 \\
{[0.386,0.816]}\end{array}$ & $31-36$ \\
\hline
\end{tabular}

Global estimate (median of posterior distribution) and $95 \%$ credible intervals (CrI) of sensitivity and specificity of tests assessing cognitive domains in subgroup of studies. $\mathrm{N}$ indicates the number of studies included in each category.

${ }^{a}$ For these data the second prior distribution analysis failed
0.539-0.738), whereas it was very high $(0.852 ; 95 \% \mathrm{CrI}$ : $0.703-0.943)$ for the three studies with the shortest followup (12 to 24 months: Eckerstrom et al. 2013; Ahmed et al. 2008; Galton et al. 2005). The second prior distribution of this meta-analysis failed and thus this result should be interpreted with caution.

There was a positive association between the length of follow-up and sensitivity values for tests of semantic fluency (0.143, 95\% CrI: 0.035-0.261: Gallagher et al. 2010; Galton et al. 2005; Ahmed et al. 2008; Ewers et al. 2012; Sarazin et al. 2007; Venneri et al. 2011). Longer mean follow-up was associated with higher sensitivity values. When examining sensitivity in the length of follow-up subgroups (Table 7), the three studies with the longest follow-up (31 to 36 months: Gallagher et al. 2010; Sarazin et al. 2007; Venneri et al. $2011)$ showed a very high sensitivity value $(0.842 ; 95 \% \mathrm{CrI}$ : 0.569-0.966), whereas those with the shortest follow-up (12 to 24 months) showed a low combined sensitivity of 0.540 (95\% CrI: 0.324-0 .722: Galton et al. 2005; Ahmed et al. 2008; Ewers et al. 2012). There was no effect of the length of follow-up for any of the other domains. Furthermore, there was no effect of age on sensitivity or specificity values.

\section{Discussion}

Neuropsychological assessment is central to the diagnosis of dementia and identifying individuals who may be in a prodromal phase of AD. People with MCI have a ten-fold higher risk of progressing to dementia every year than people of the same age in the general population. Thus, many people with MCI are actually in a prodromal stage of AD. However, not all patients with MCI progress to dementia and it is thus critical for clinicians to identify tools that can accurately separate those who will remain stable from those who will progress to dementia. Many longitudinal studies have published predictive accuracy values for different cognitive tests. There is a critical need for a systematic analysis of this literature because of the large number of cognitive dimensions that can be measured and because each domain can be assessed with still a larger number of neuropsychological tools.

In this systematic review, we found 28 longitudinal studies that assessed the values of neuropsychological tests to predict progression from MCI to dementia. We selected studies based on strict inclusion and exclusion criteria and hence discarded studies that contained fatal methodological flaws for a metaanalysis of predictive diagnostic test accuracy (for example, those that failed to use clinical criteria to identify progression to dementia or those for which the methodological information provided was insufficient to allow replication). Nevertheless, it was important to assess the general quality level of the remaining studies and whether the methodology could bias the data, as the studies varied somewhat. Based on the quality criteria used here (QUADAS tool and Cochrane guidelines), most studies had a relatively low risk of bias. Furthermore, the studies were relatively homogenous in their methodological approach and relied on well-accepted clinical criteria to identify their patients and outcomes. Most included studies relied on a prospective design and used Petersen's criteria to select their participants, and all relied on the NINCDS-ARDRA to identify dementia. Yet, 10 of the 28 studies showed a high risk of bias. A few features were found to be problematic. The most frequent problem was related to the selection process, which led to the sample not being representative of the population of interest. This limitation might have a negative impact on the generalizibility of the results from these studies. Another problem was related to a failure to keep predictive tests independent from the gold standard used to identify the outcome (here, progression to dementia). This was found in five studies, and five others failed to report information regarding this criterion. This is an important methodological control because predictive accuracy can be artificially inflated when the predictors are not kept independent from the standard. 
We decided to focus on measures of sensitivity and specificity and to report both independently rather than focusing mostly on overall predictive accuracy. Ideally, a test with optimal predictive accuracy should combine excellent sensitivity and specificity. However, the optimal ratio of specificity to sensitivity may also depend on the clinical and research context. For example, clinicians might favor sensitivity over specificity to detect a deadly disease that could be cured if treated. In contrast, specificity might be favored over sensitivity when a disease cannot be treated, when a diagnosis has the potential to result in stigmatization, exclusion or depression, or when treatment has important side effects. In a research context, investigators might favor a different balance between sensitivity and specificity as a function of their research question. Remarkably, our systematic review indicated that many domains and tests show an appropriate balance with very good sensitivity and specificity values.

The studies that we reviewed covered data for a total of 2365 participants who met the criteria for MCI at entry and were followed over an average of 31 months to assess whether they met the criteria for AD type dementia. In total, 916 individuals with $\mathrm{MCI}$ were later found to progress to dementia. This represents a progression rate of $38.7 \%$, which is fairly consistent with the literature, considering the 31-month average follow-up (Gauthier et al. 2006). However, the progression rate across individual studies was quite variable, ranging from 6 to $39 \%$ per year.

The systematic review examined 61 cognitive tests that evaluated 22 cognitive dimensions. It identified many neuropsychological measures with very good sensitivity for predicting dementia, with some reaching more than $90 \%$. Similarly, many neuropsychological tests revealed excellent specificity values. Five neuropsychological measures had an overall accuracy of greater than $90 \%$. Three were episodic memory tests (Guild paragraph delayed recall, RAVLT delayed recall, and free delayed recall of names from a facename association) and two measured visual semantics (object function recognition and the VOSP silhouette). One global test measuring different cognitive components (ACE Addenbrooke's cognitive examination) also yielded excellent overall accuracy. Thus, although the sensitivity to specificity ratio varied for individual tests, many had an appropriate balance between the two and some showed both excellent sensitivity and specificity. This systematic review also examined the predictive value of studies that examined a combination of cognitive measures. The use of a combination of neuropsychological measures is likely to be the best approach to identify future progressors, because the sensitivity to specificity ratio varies for individual domains. Studies that have examined combined markers generally reported very high to excellent predictive accuracy with a good balance between sensitivity and specificity, particularly when they combined memory with executive or language tests.
We performed a meta-analysis of the predictive accuracy for 14 cognitive domain categories that included at least three independent studies. The meta-analysis pooled sensitivity and specificity values to obtain quantitative indicators. The metaanalysis showed that most measures of verbal memory were excellent predictors with very good $(\geq 0.7)$ specificity and sensitivity values. In addition, predictive values from verbal memory tests were barely influenced by the testing conditions. For example, delayed recall did not predict progression better than immediate recall. Similarly, there was no major difference between cued recall and free recall and there was no added benefit from providing orientation at retrieval. This goes against the concept that measures of delayed recall or tests that orient processing at encoding are the best indicators of early $\mathrm{AD}$, because they reflect hippocampal dysfunction (Dubois et al. 2007; Albert et al. 2011). The present data indicate rather that a range of verbal memory tests can be used as appropriate indicators of early $\mathrm{AD}$ and that the nature of the task may not profoundly influence predictive accuracy. Contrary to the general finding of high predictive value for verbal memory tasks, two testing conditions were associated with relatively low predictive accuracy: word recognition and word recall with orientation at encoding and cues at retrieval. This is consistent with the notion that $\mathrm{AD}$ patients suffer from impaired encoding, because being impaired on tasks that increases encoding is not a good predictor of progression.

Interestingly, some language categories were rather good predictors of future decline, particularly naming and semantic fluency. These tests implicate semantic memory and some form of executive functions, which may explain their ability to predict future decline, as both processes were proposed to be impaired early in MCI (Belleville et al. 2008; Joubert et al. 2008). The predictive accuracy of two language tests (fluency and naming) was modified as a function of the length of followup. This indicates that the predictive value of language categories varies with disease stage in the prodromal continuum, contrary to verbal memory, for which predictive accuracy was similar, irrespective of the prodromal stage of the patient. This finding and its implications will be discussed further below.

Several cognitive categories showed better specificity than sensitivity values. This was true for memory tasks that provide support at encoding and retrieval, for example, recognition ( 0.547 and 0.789 for sensitivity and specificity, respectively) or word-list cued delayed recall with oriented encoding $(0.676$ and 0.896 for sensitivity and specificity, respectively). The same pattern was found for some non-memory categories as well. For example shifting (sensitivity $=0.541$; specificity $=0.679$ ), working memory (sensitivity $=0.599$; specificity $=0.667$ ), and semantic knowledge (sensitivity $=0.703$; specificity $=0.814$ ) showed higher specificity than sensitivity. Thus, these tests may not be very sensitive to identify future progression, but they might be useful for identifying patients 
with MCI who will remain stable and thus contribute to reduce the number of false positives.

The meta-regressions examined whether age and length of follow-up determined differences in sensitivity and specificity values. Age had no effect on predictive accuracy. However, conclusions concerning age might be limited by the fact that this aspect was examined across studies and not across individuals. It is therefore possible that the search for an effect was limited by the lack of difference in the average age across studies, because disease onset is likely to be equivalent across different samples. The effect of the length of follow-up is perhaps more informative, as studies have control over this variable, which differed between studies. The predictive accuracy of naming and the semantic fluency category varied as a function of the length of follow-up. Studies with very short follow-ups might increase the likelihood of false negatives, as they do not allow sufficient time for individuals to progress to meet the dementia criteria. It is unlikely that a test would be more sensitive for a shorter than longer follow-up, as symptoms increase with progression. Thus, higher sensitivity for studies with longer follow-ups might reflect such a phenomenon. Semantic fluency followed this pattern. Fluency tasks yielded excellent sensitivity (0.842) for long follow-ups (31 to 36 months), but sensitivity markedly declined (0.540) for short follow-ups (12 to 24 months). Hence, this interaction might reflect the contribution of false negative cases to the data from studies relying on short follow-ups.

Assessing the impact of the length of follow-up is also informative for identifying very early markers. Hence, a reasonably long follow-up can be used as a proxy for how far the patient was from the diagnosis when the test was given. Determining what constitutes a reasonable follow-up is complex, but if approximately $15 \%$ of individuals with MCI progress to dementia yearly, and approximately $25 \%$ of them remain stable, irrespective of follow-up length, a three-year follow-up would allow the detection of approximately $60 \%$ of the MCI progressors, which would increase to $80 \%$ for a fouryear follow-up. A task found to be predictive, irrespective of the length of follow-up, is a good candidate for an early predictor. A task found to be less predictive at a longer than shorter follow-up may be less well-suited as an early indicator of future dementia and more representative of imminent decline. This pattern was found for the naming category. Although the test showed good specificity at longer followups ( 0.648 for follow-ups of 31 to 36 months), specificity was markedly increased, and excellent, at shorter follow-ups ( 0.852 for follow-ups of 12 to 24 months). Sensitivity was unaffected by the length of follow-up, suggesting that naming might fare better as a predictor at shorter rather than longer follow-up and might be better used as a marker of imminent progression rather than an early marker of the disease. This result and its interpretation needs to be confirmed by future studies and meta-analyses, because the effect was not found when examining the alternative distribution and it was based on a relatively small number of studies.

One strength of this meta-analysis was the use of the Bayesian approach, which has several advantages over other frequently used approaches. Simulation studies have shown that the Bayesian method provides better coverage probabilities for global sensitivity and specificity, particularly in the case of sparse data (Paul et al. 2010). One reason for this is that Bayesian inferences generally come with wider credibility intervals (often more realistic) than frequently used alternative methods (Warn et al. 2002). The Bayesian approach yields less biased estimations of variance and correlation parameters (Paul et al. 2010). Other frequently used approaches often experience convergence issues, which are less of an issue with the Bayesian approach (Paul et al. 2010). Finally, the Bayesian approach generally produces an approximate joint posterior distribution of all model parameters. This has the advantage of not only making it possible to test hypotheses, but also to obtain the probability that any given parameter is above or below any given threshold (Rutter and Gatsonis 2001). It also allows the easy computation of point estimates of any functions of Se and $\mathrm{Sp}$, such as predictive values or likelihood ratios, along with their credibility intervals.

This study has limitations. One major limitation is that we focused on individuals meeting the criteria for MCI, which might not represent the earliest stage of AD. Thus, the follow-up periods reported in the included studies are relatively short if one considers that the disease develops over two decades prior to diagnosis. Therefore, it is unclear whether the predictive accuracy identified here is representative of earlier stages and whether it can be extended further back during the prodromal period. It is also possible that a slightly different ensemble of tasks would display different sensitivity and specificity values at an earlier period. Future studies could metaanalyze data from individuals reporting a subjective cognitive decline to assess earlier markers, as these individuals might be in a phase that precedes MCI in the disease continuum (Jessen et al. 2014). Similarly, the outcome of studies interested in predementia diagnosis depends on the type of recruitment at entry and the validity of the classification scheme, for example, how subjective cognitive decline or MCI is diagnosed. Advances in the field and refinement of diagnostic criteria will certainly increase the ability to identify early markers. Another limitation is the large variability in the tasks that were tested across studies. As a result, we focused on cognitive domain categories rather than individual tasks for the meta-analyses. Although this quantitative meta-analysis provides information as to the domains that should be measured for early prediction, it does not identify specific tests. However, the systematic review included in this study identifies the predictive accuracy for a range of neuropsychological tests that map the cognitive domain categories identified in the meta-analysis. Our finding that the use of different testing conditions for memory tasks 
does not substantially modify predictive accuracy lends support to our approach, as it indicates that sensitivity and specificity do not vary much within broad cognitive domains. As already mentioned, the statistical analyses were limited by the small number of included studies. This does not diminish the reliability of the results, but the high between-study variability was reflected by the large CrI widths. Some statistical assumptions, for example that of normality, could not be verified due to the small number of studies. Also, the reported CrIs are not applicable to a hypothetical future study. For example, using different cut-offs would change the sensitivity and specificity values. We experienced instances of convergence failures. The Bayesian approach that we used is known to be less prone to convergence issues than other frequently used methods (Paul et al. 2010). Yet, when there are relatively few studies with sparse data, convergence can be particularly difficult to achieve because it may lead to very wide estimations of the corresponding credibility intervals. This is even more challenging for meta-regression models as they contain additional parameters. We suspect that the convergence failures were due to small amounts of data compared to the number of model parameters. Finally, sensitivity and specificity values were slightly modified in four cases after excluding studies with a high risk of bias (reduced sensitivity for three, increased specificity for one). In these cases, correct inferences for sensitivities and specificities probably fell somewhere between the original results (left-hand side of Table 5) and those excluding high-risk studies (right-hand side of Table 5).

In conclusion, the results from this meta-analysis are encouraging for those interested in the early identification of AD. They show that neuropsychological assessment, which is affordable and widely accessible, can strongly contribute to predicting dementia while individuals are still in the MCI phase. The meta-analysis revealed very good to excellent predictive accuracy for many cognitive domains, particularly those concerned with verbal memory and semantic processing. Based on the meta-analyzed data, performance on cognitive tests can predict whether MCI patients will progress to dementia at least 3 years prior to the time at which the diagnosis is made and should contribute highly to the development of early indices of AD.

Acknowledgements The study is part of the Quebec Consortium for the early identification of Alzheimer's disease (CIMA-Q) funded by the Fonds d'Innovation Pfizer-FRQS and Quebec Network for Research on Aging (RQRV) of the Fonds de Recherche du Québec-Santé. The funding agencies had no role in the process or content of the meta-analysis. We would like to thank the CIMA-Q Groupe Cognition (Ana Ines Ansaldo, Marie-André Bruneau, Jean-François Gagnon, Isabelle Rouleau, Natasha Rajah, Howard Chertkow, Natalie Philips, Dominique Lorrain, Stéphane Poulin, Simona Brambati, Sébastien Grenier, and Thanh Dang Vu) for their contribution in identifying cognitive domain categories. We would also like to thank Christine St-Pierre for her advice on methodology, Isabel Arsenault for help in formatting the last version of the manuscript,
Audrey Attia for her support in defining the search strategy, and Gabrielle Ciquier Sell and Alex Edelman and Associates for revision of the English.

\section{Compliance with Ethical Standards}

Conflicts of Interest S. Belleville receives research grants from the Fonds d'Innovation Pfizer-FRQS for Alzheimer's disease and related diseases for the Consortium pour l'identification Précoce de la Maladie d'Alzheimer - Québec (CIMA-Q). She has been a consultant for research development on the prevention of Alzheimer's Disease for the Fondation IUGM (2016) and a consultant for the development of a cognitive stimulation program for the Centre de Promotion de la Santé AvantÂge (2015). She has intellectual property rights for the Programme de Stimulation pour une santé cognitive, Memoria, Batterie d'évaluation de la mémoire Côte-des-Neiges and MEMO, Programme pour une mémoire optimale.

C. Hudon receives funding from the Fonds d'Innovation Pfizer-FRQS for Alzheimer's disease and related diseases for the Consortium pour l'identification Précoce de la Maladie d'Alzheimer - Québec (CIMA-Q) and from the Canadian Institutes on Health Research (MOP-119612). He also received a salary award (Chercheur-boursier Senior) from the FRQS. He received honoraria as a speaker at a Lundbeck Colloquium held in Québec City (March 2016). He is a consultant for Cogstate, Bracket and a rater for Roche and Lundbeck.

C. Fouquet, H.T.V Zomahoun, and J. Croteau report no conflict of interest.

Open Access This article is distributed under the terms of the Creative Commons Attribution 4.0 International License (http:// creativecommons.org/licenses/by/4.0/), which permits unrestricted use, distribution, and reproduction in any medium, provided you give appropriate credit to the original author(s) and the source, provide a link to the Creative Commons license, and indicate if changes were made.

\section{References}

Ahmed, S., Mitchell, J., Arnold, R., Nestor, P. J., \& Hodges, J. R. (2008). Predicting rapid clinical progression in amnestic mild cognitive impairment. Dementia and Geriatric Cognitive Disorders, 25(2), 170 $177 \mathrm{https}: / /$ doi.org/10.1159/000113014.

Albert, M. S., DeKosky, S. T., Dickson, D., Dubois, B., Feldman, H. H., Fox, N. C., et al. (2011). The diagnosis of mild cognitive impairment due to Alzheimer's disease: Recommendations from the National Institute on Aging-Alzheimer's Association workgroups on diagnostic guidelines for Alzheimer's disease. Alzheimers Dement, 7(3), 270-279 https://doi.org/10.1016/j.jalz.2011.03.008.

Albert, M. S., Moss, M. B., Tanzi, R., \& Jones, K. (2001). Preclinical prediction of $\mathrm{AD}$ using neuropsychological tests. Journal of the International Neuropsychological Society, 7(5), 631-639 https:// doi.org/10.1017/S1355617701755105.

Amieva, H., Le Goff, M., Millet, X., Orgogozo, J. M., Peres, K., Barberger-Gateau, P., et al. (2008). Prodromal Alzheimer's disease: Successive emergence of the clinical symptoms. Annals of Neurology, 64(5), 492-498 https://doi.org/10.1002/ana.21509.

Anchisi, D., Borroni, B., Franceschi, M., Kerrouche, N., Kalbe, E., Beuthien-Beumann, B., et al. (2005). Heterogeneity of brain glucose metabolism in mild cognitive impairment and clinical progression to Alzheimer disease. [Comparative Study Evaluation Studies Research Support, Non-U.S. Gov't]. Archives of Neurology, 62(11), 1728-1733.

Arnaiz, E., Jelic, V., Almkvist, O., Wahlund, L. O., Winblad, B., Valind, S., et al. (2001). Impaired cerebral glucose metabolism and 
cognitive functioning predict deterioration in mild cognitive impairment. Neuroreport, 12(4), 851-855.

Association, A. P. (1987). Diagnostic and statistical manual of mental disorders, ed 3, rev. Washington, DC: American Psychiatric Association.

Association, A. P. (1994). Diagnostic and statistical manual of mental disorders (4th ed.). Washington, DC: American Psychiatric Association.

Association, A. P. (2013). Diagnostic and statistical manual of mental disorders (DSM-5®) (5th ed.). Washington, DC: American Psychiatric Publishing, Inc.

Babins, L., Slater, M. E., Whitehead, V., \& Chertkow, H. (2008). Can an 18-point clock-drawing scoring system predict dementia in elderly individuals with mild cognitive impairment? Journal of Clinical \& Experimental Neuropsychology: Official Journal of the International Neuropsychological Society, 30(2), 173-186 https:// doi.org/10.1080/13803390701336411.

Bateman, R. J., Xiong, C., Benzinger, T. L., Fagan, A. M., Goate, A., Fox, N. C., et al. (2012). Clinical and biomarker changes in dominantly inherited Alzheimer's disease. The New England Journal of Medicine, 367(9), 795-804 https://doi.org/10.1056/ NEJMoa1202753.

Belleville, S., Chertkow, H., \& Gauthier, S. (2007). Working memory and control of attention in persons with Alzheimer's disease and mild cognitive impairment. [Research Support, Non-U.S. Gov't]. Neuropsychology, 21(4), 458-469 https://doi.org/10.1037/08944105.21.4.458.

Belleville, S., Fouquet, C., Duchesne, S., Collins, D. L., \& Hudon, C. (2014a). Detecting early preclinical Alzheimer's disease via cognition, neuropsychiatry, and neuroimaging: Qualitative review and recommendations for testing. [Research Support, Non-U.S. Gov't]. Journal of Alzheimer's Disease, 42(Suppl 4), S375-S382 https://doi. org/10.3233/JAD-141470.

Belleville, S., Gauthier, S., Lepage, E., Kergoat, M. J., \& Gilbert, B. (2014b). Predicting decline in mild cognitive impairment: A prospective cognitive study. Neuropsychology, 28(4), 643-652 https:// doi.org/10.1037/neu0000063.

Belleville, S., Sylvain-Roy, S., de Boysson, C., \& Menard, M. C. (2008). Characterizing the memory changes in persons with mild cognitive impairment. [Research Support, Non-U.S. Gov't review]. Progress in Brain Research, 169, 365-375 https://doi.org/10.1016/S00796123(07)00023-4.

Buchhave, P., Stomrud, E., Warkentin, S., Blennow, K., Minthon, L., \& Hansson, O. (2008). Cube copying test in combination with $\mathrm{rCBF}$ or CSF a beta(42) predicts development of Alzheimer's disease. Dementia and Geriatric Cognitive Disorders, 25(6), 544-552.

Dahabreh, I. J., Trikalinos, T. A., Lau, J., \& Schmid, C. (2012). An empirical assessment of bivariate methods for meta-analysis of test accuracy. Methods Research Report. (Prepared by Tufts Evidencebased Practice Center under Contract No. 290-2007-10055-I.) AHRQ Publication No 12(13)-EHC136-EF. Rockville: Agency for Healthcare Research and Quality. November 2012. www. effectivehealthcare.ahrq.gov/reports/final/cfm.

Defrancesco, M., Marksteiner, J., Deisenhammer, E., Kemmler, G., Djurdjevic, T., \& Schocke, M. (2013). Impact of white matter lesions and cognitive deficits on conversion from mild cognitive impairment to alzheimer's disease. Journal of Alzheimer's Disease, 34(3), 665-672 https://doi.org/10.3233/JAD-122095.

Didic, M., Felician, O., Barbeau, E. J., Mancini, J., Latger-Florence, C., Tramoni, E., et al. (2013). Impaired visual recognition memory predicts Alzheimer's disease in amnestic mild cognitive impairment. Dementia and Geriatric Cognitive Disorders, 35(5-6), 291-299 https://doi.org/10.1159/000347203.

Dierckx, E., Engelborghs, S., De Raedt, R., Van Buggenhout, M., De Deyn, P. P., Verte, D., et al. (2009). Verbal cued recall as a predictor of conversion to Alzheimer's disease in mild cognitive impairment. International Journal of Geriatric Psychiatry, 24(10), 1094-1100.
Dubois, B., Feldman, H. H., Jacova, C., Dekosky, S. T., BarbergerGateau, P., Cummings, J., et al. (2007). Research criteria for the diagnosis of Alzheimer's disease: Revising the NINCDS-ADRDA criteria. Lancet Neurology, 6(8), 734-746 https://doi.org/10.1016/ S1474-4422(07)70178-3.

Eckerstrom, C., Olsson, E., Bjerke, M., Malmgren, H., Edman, A., Wallin, A., et al. (2013). A combination of neuropsychological, neuroimaging, and cerebrospinal fluid markers predicts conversion from mild cognitive impairment to dementia. Journal of Alzheimer's Disease, 36(3), 421-431 https://doi.org/10.3233/JAD-122440.

Ewers, M., Walsh, C., Trojanowski, J. Q., Shaw, L. M., Petersen, R. C., Jack Jr., C. R., et al. (2012). Prediction of conversion from mild cognitive impairment to Alzheimer's disease dementia based upon biomarkers and neuropsychological test performance. [Research Support, N.I.H., Extramural Research Support, Non-U.S. Gov't]. Neurobiology of Aging, 33(7), 1203-1214 https://doi.org/10.1016/ j.neurobiolaging.2010.10.019.

Flicker, C., Ferris, S. H., \& Reisberg, B. (1991). Mild cognitive impairment in the elderly: Predictors of dementia. [Research Support, U.S. Gov't, P.H.S.] Neurology, 41(7), 1006-1009.

Gallagher, D., Mhaolain, A. N., Coen, R., Walsh, C., Kilroy, D., Belinski, K., et al. (2010). Detecting prodromal Alzheimer's disease in mild cognitive impairment: Utility of the CAMCOG and other neuropsychological predictors. International Journal of Geriatric Psychiatry, 25(12), 1280-1287 https://doi.org/10.1002/gps.2480.

Galton, C. J., Erzinclioglu, S., Sahakian, B. J., Antoun, N., \& Hodges, J. R. (2005). A comparison of the Addenbrooke's cognitive examination (ACE), conventional neuropsychological assessment, and simple MRI-based medial temporal lobe evaluation in the early diagnosis of Alzheimer's disease. [Comparative Study Research Support, Non-U.S. Gov't]. Cognitive and Behavioral Neurology, 18(3), 144150.

Gauthier, S., Patterson, C., Gordon, M., Soucy, J. P., Schubert, F., \& Leuzy, A. (2011). Commentary on "recommendations from the National Institute on Aging-Alzheimer's Association workgroups on diagnostic guidelines for Alzheimer's disease." a Canadian perspective. Alzheimers Dement, 7(3), 330-332 https://doi.org/10. 1016/j.jalz.2011.03.006.

Gauthier, S., Reisberg, B., Zaudig, M., Petersen, R. C., Ritchie, K., Broich, K., et al. (2006). Mild cognitive impairment. Lancet, 367(9518), 1262-1270.

Griffith, H. R., Netson, K. L., Harrell, L. E., Zamrini, E. Y., Brockington, J. C., \& Marson, D. C. (2006). Amnestic mild cognitive impairment: Diagnostic outcomes and clinical prediction over a two-year time period. Journal of the International Neuropsychological Society, 12(2), 166-175 https://doi.org/10.1017/S1355617706060267.

Guo, J., \& Riebler, A. (2016). R package meta4diag: meta-analysis for diagnostic test studies. Trondheim: Norwegian University of Science and Technology.

Haynes, R. B., Sackett, D. L., Guyatt, G. H., \& Tugwell, P. (2006). Clinical epidemiology: How to do clinical practice research (3rd ed.). Philadelphia: Lippincott Williams \& Wilkins.

HIQA - IRELAND, IQWiG - GERMANY. (2015). Meta-analysis of Diagnostic Test Accuracy Studies. Eunethta. http://www.eunethta. eu/outputs/methodological-guideline-meta-analysis-diagnostic-testaccuracystudies.Accessed 14 Sep 2017.

Irish, M., Lawlor, B. A., Coen, R. F., \& O'Mara, S. M. (2011). Everyday episodic memory in amnestic mild cognitive impairment: A preliminary investigation. BMC Neuroscience, 12, $80 \mathrm{https} / / / \mathrm{doi} .0 \mathrm{rg} / 10$. 1186/1471-2202-12-80.

Jessen, F., Wolfsgruber, S., Wiese, B., Bickel, H., Mosch, E., Kaduszkiewicz, H., et al. (2014). AD dementia risk in late MCI, in early MCI, and in subjective memory impairment. [Research Support, Non-U.S. Gov't]. Alzheimers Dement, 10(1), 76-83 https://doi.org/10.1016/j.jalz.2012.09.017. 
Joubert, S., Felician, O., Barbeau, E. J., Didic, M., Poncet, M., \& Ceccaldi, M. (2008). Patterns of semantic memory impairment in mild cognitive impairment. Behavioural Neurology, 19(1-2), 3540.

Kluger, A., Ferris, S. H., Golomb, J., Mittelman, M. S., \& Reisberg, B. (1999). Neuropsychological prediction of decline to dementia in nondemented elderly. [Research Support, U.S. Gov't, P.H.S.] Journal of Geriatric Psychiatry and Neurology, 12(4), 168-179.

Leeflang, M. G. G. (2014). Systematic reviews and meta-analyses of diagnostic test accuracy. Clinical Microbiology and Infection (20), 105-113.

Lekeu, F., Magis, D., Marique, P., Delbeuck, X., Bechet, S., Guillaume, B., et al. (2010). The California verbal learning test and other standard clinical neuropsychological tests to predict conversion from mild memory impairment to dementia. Journal of Clinical and Experimental Neuropsychology, 32(2), 164-173 https://doi.org/10. 1080/13803390902889606.

Liberati, A., Altman, D. G., Tetzlaff, J., Mulrow, C., Gotzsche, P. C., Ioannidis, J. P., et al. (2009). The PRISMA statement for reporting systematic reviews and meta-analyses of studies that evaluate health care interventions: Explanation and elaboration. [Consensus Development Conference Guideline Research Support, Non-U.S. Gov't]. Journal of Clinical Epidemiology, 62(10), e1-34 https:// doi.org/10.1016/j.jclinepi.2009.06.006.

Lischka, A. R., Mendelsohn, M., Overend, T., \& Forbes, D. (2012). A systematic review of screening tools for predicting the development of dementia. Canadian Journal on Aging, 31(3), 295-311 https:// doi.org/10.1017/S0714980812000220.

Macaskill, P., Gatsonis, C., Deeks, J. J., Harbord, R. M., \& Takwoingi, Y. I. (2010.). Chapter 10: Analysing and presenting results. In J. J. Deeks, P. M. Bossuyt, \& C. Gatsonis (Eds.), Cochrane Handbook for Systematic Reviews of Diagnostic Test Accuracy Version 1.0. Available from: http://Srdta.Cochrane.Org/. The Cochrane collaboration.

Marcos, A., Gil, P., Barabash, A., Rodriguez, R., Encinas, M., Fernandez, C., et al. (2006). Neuropsychological markers of progression from mild cognitive impairment to Alzheimer's disease. [Research Support, Non-U.S. Gov't]. American Journal of Alzheimer's Disease and Other Dementias, 21(3), 189-196.

Mattis, S. (1988). Dementia rating scale. Odessa: Psychological Assessment Resources.

McKhann, G., Drachman, D., Folstein, M., Katzman, R., Price, D., \& Stadlan, E. M. (1984). Clinical diagnosis of Alzheimer's disease: Report of the NINCDS-ADRDA work group under the auspices of Department of Health and Human Services Task Force on Alzheimer's disease. Neurology, 34(7), 939-944.

McKhann, G., Knopman, D. S., Chertkow, H., Hyman, B. T., Jack Jr., C. R., Kawas, C. H., et al. (2011). The diagnosis of dementia due to Alzheimer's disease: Recommendations from the National Institute on Aging-Alzheimer's Association workgroups on diagnostic guidelines for Alzheimer's disease. Alzheimers Dement, 7(3), 263-269 https://doi.org/10.1016/j.jalz.2011.03.005.

Mitchell, J., Arnold, R., Dawson, K., Nestor, P. J., \& Hodges, J. R. (2009). Outcome in subgroups of mild cognitive impairment (MCI) is highly predictable using a simple algorithm. [Research Support, Non-U.S. Gov't]. Journal of Neurology, 256(9), 1500 $1509 \mathrm{https}: / /$ doi.org/10.1007/s00415-009-5152-0.

Ozer, S., Young, J., Champ, C., \& Burke, M. (2016). A systematic review of the diagnostic test accuracy of brief cognitive tests to detect amnestic mild cognitive impairment. International Journal of Geriatric Psychiatry, 31(11), 1139-1150 https://doi.org/10.1002/ gps.4444.

Paul, M., Riebler, A., Bachmann, L. M., Rue, H., \& L., H. (2010). Bayesian bivariate meta-analysis of diagnostic test studies using integrated nested Laplace approximations. Statistics in Medicine, 29(12), 1325-1339.
Perri, R., Serra, L., Carlesimo, G. A., Caltagirone, C., \& Early Diagnosis Group of Italian Interdisciplinary Network on Alzheimer's, D. (2007a). Preclinical dementia: An Italian multicentre study on amnestic mild cognitive impairment. [Multicenter Study]. Dementia and Geriatric Cognitive Disorders, 23(5), 289-300.

Perri, R., Serra, L., Carlesimo, G. A., Caltagirone, C., \& Early Diagnosis Group of the Italian Interdisciplinary Network on Alzheimer's, D. (2007b). Amnestic mild cognitive impairment: Difference of memory profile in subjects who converted or did not convert to Alzheimer's disease. Neuropsychology, 21(5), 549-558 https://doi. org/10.1037/0894-4105.21.5.549.

Petersen, R. C. (2000). Mild cognitive impairment: Transition between aging and Alzheimer's disease. [Research Support, U.S. Gov't, P.H.S.] Neurología, 15(3), 93-101.

Reitsma, J., Rutjes, A. W., Whiting, P., Vlassov, V. V., Leeflang, M. M., \& Deeks, J. J. (2009). Chapter 9: Assessing methodological quality. In J. J. Deeks, P. M. Bossuyt \& C. Gatsonis (Eds.), The Cochrane Collaboration. http://srdta.cochrane.org/

Reitsma, J. B., Glas, A. S., Rutjes, A. W., Scholten, R. J., Bossuyt, P. M., \& Zwinderman, A. H. (2005). Bivariate analysis of sensitivity and specificity produces informative summary measures in diagnostic reviews. [Review]. Journal of Clinical Epidemiology, 58(10), 982-990 https://doi.org/10.1016/j.jclinepi.2005.02.022.

Richard, E., Schmand, B. A., Eikelenboom, P., \& Van Gool, W. A. (2013). MRI and cerebrospinal fluid biomarkers for predicting progression to Alzheimer's disease in patients with mild cognitive impairment: A diagnostic accuracy study. BMJ Open, 3(6). https://doi. org/10.1136/bmjopen-2012-002541.

Robert, C. P. (2001). Chapter 5. In The Bayesian choice: from decisiontheoretic foundations to computational implementation. New York: Springer.

Rue, H., Martino, S., \& Chopin, N. (2009). Approximate Bayesian inference for latent Gaussian models using integrated nested Laplace approximations. Journal of the Royal Statistical Society B (71), 319-392.

Rutter, C. M., \& Gatsonis, C. A. (2001). A hierarchical regression approach to meta-analysis of diagnostic test accuracy evaluations. Statistics in Medicine, 20(19), 2865-2884.

Sarazin, M., Berr, C., De Rotrou, J., Fabrigoule, C., Pasquier, F., Legrain, S., et al. (2007). Amnestic syndrome of the medial temporal type identifies prodromal AD: A longitudinal study. [Erratum appears in neurology. 2008 May 20;70(21):2016]. [Comparative Study Research Support, Non-U.S. Gov't]. Neurology, 69(19), 1859-1867.

Saunders, N. L., \& Summers, M. J. (2010). Attention and working memory deficits in mild cognitive impairment. Journal of Clinical and Experimental Neuropsychology, 32(4), 350-357 https://doi.org/10. 1080/13803390903042379.

Spiegelhalter, D. J., Myles, J. P., Jones, D. R., \& Abrams, K. R. (1999). An introduction to Bayesian methods in health technology assessment. BMJ, 319(7208), 508-512.

Summers, M. J., \& Saunders, N. L. (2012). Neuropsychological measures predict decline to Alzheimer's dementia from mild cognitive impairment. Neuropsychology, 26(4), 498-508.

Tabert, M. H., Manly, J. J., Liu, X., Pelton, G. H., Rosenblum, S., Jacobs, M., et al. (2006). Neuropsychological prediction of conversion to alzheimer disease in patients with mild cognitive impairment. Archives of General Psychiatry, 63(8), 916-924 https://doi.org/10. 1001/archpsyc.63.8.916.

Tierney, M. C., Szalai, J. P., Snow, W. G., Fisher, R. H., Nores, A., Nadon, G., et al. (1996). Prediction of probable Alzheimer's disease in memory-impaired patients: A prospective longitudinal study. [Research Support, Non-U.S. Gov't]. Neurology, 46(3), 661-665.

Tsoi, K. K., Chan, J. Y., Hirai, H. W., Wong, S. Y., \& Kwok, T. C. (2015). Cognitive tests to detect dementia: A systematic review and metaanalysis. JAMA Internal Medicine, 175(9), 1450-1458 https://doi. org/10.1001/jamainternmed.2015.2152. 
Venneri, A., Gorgoglione, G., Toraci, C., Nocetti, L., Panzetti, P., \& Nichelli, P. (2011). Combining neuropsychological and structural neuroimaging indicators of conversion to alzheimer's disease in amnestic mild cognitive impairment. Current Alzheimer Research, 8(7), 789-797 https://doi.org/10.2174/156720511797633160.

Visser, P. J., Verhey, F. R. J., Ponds, R., \& Jolles, J. (2001). Diagnosis of preclinical Alzheimer's disease in a clinical setting. [Article]. International Psychogeriatrics, 13(4), 411-423.

Whiting, P., Rutjes, A. W., Reitsma, J. B., Bossuyt, P. M., \& Kleijnen, J. (2003). The development of QUADAS: A tool for the quality assessment of studies of diagnostic accuracy included in systematic reviews. [Evaluation Studies Research Support, Non-U.S. Gov't].
BMC Medical Research Methodology, 3, 25, https://doi.org/10. 1186/1471-2288-3-25.

Warn, D. E., Thompson, S. G., \& Spiegelhalter, D. J. (2002). Bayesian random effects meta-analysis of trials with binary outcomes: Methods for the absolute risk difference and relative risk scales. Statistics in Medicine, 21(11), 1601-1623.

Whiting, P. F., Rutjes, A. W., Westwood, M. E., Mallett, S., Deeks, J. J., Reitsma, J. B., et al. (2011). QUADAS-2: A revised tool for the quality assessment of diagnostic accuracy studies. [Research Support, Non-U.S. Gov't]. Annals of Internal Medicine, 155(8), 529-536 https://doi.org/10.7326/0003-4819-155-8-20111018000009 . 